\title{
COMPARISON OF SOME INTER-CELL INTERFERENCE MOdEls For CELlular NETWORKS
}

\author{
Olav N. Østerbø and Ole Grøndalen \\ Telenor Research, Telenor ASA, Oslo, Norway
}

\begin{abstract}
In this paper we discuss and compare methods to analyse the influence inter-cell interference will have on coverage/outage probabilities in cellular networks. The framework is based on a common method to find the Laplace transform of the distribution of interference from neighbouring cells. It turns out that for Suzuki distributed fading the analysis is highly simplified. In case of Rayleigh faded channels only, the analysis is even more simplified. The modelling approach, which is based on classical probability methods, rather than on modern measure theory for point processes, allows for both fixed and stochastic locations of base stations. The different models are applied to quantify the effect of inter-cell interference on coverage/outage probabilities and on spectrum efficiencies in LTE networks. We consider several scenarios ranging from fixed hexagonal layout of base station to stochastic location of based on uniform distribution of base stations. We also extend the coverage/outage analysis for the Ginibre Point Process to Suzuki faded environment. Numerical examples show large differences in both spectrum efficiency and coverage/outage probabilities for the different network scenarios considered.
\end{abstract}

\section{KEYWORDS}

Cellular Networks, Outage Probability, SINR, Spectrum Efficiency

\section{INTRODUCTION}

The demand for cellular capacity is steadily increasing. In particular 4G, e.g. LTE (Long Term Evolution) and also $5 \mathrm{G}$, now standardized by $3 \mathrm{GPP}$, will boost the capacity compared to legacy mobile networks and will bring significant enhancements in terms of spectrum efficiency, peak data rate and latency. One of the main hard struggles in Orthogonal Frequency-Division Multiple Access (OFDMA) networks like LTE is to design effective mechanisms that minimise the effect of the other-cell interference; since the Signal-to-Interference-plus-Noise Ratio (SINR) which actually determines the obtainable bit rate, strongly dependent of the magnitude of this interference. Hence, accurate models that describe the other-cell interference will be important to be able to maximise the SINR for users and therefore increasing the overall capacity and improving Quality of Service (QoS) in such networks.

In the literature the modelling of other-cell inference is not very mature mainly because of the difficulties to model the interference efficiently. For downlink the interference will be the sum of signals from all surrounded base stations (BSs) using the same frequency as a tagged user. In several papers a fixed regular hexagonal cell layout is used as a basis for interference estimations. Since actual BS layouts are far from regular, a stochastic modelling may be beneficial. It turns out that if the BSs are located according to a two dimensional Poisson Point Process (PPP), the analysis is heavily simplified and closed form expression of the coverage probability can be obtained in closed form for the case of Rayleigh fading [1]. However models based on PPPs have the drawback that BSs may be placed arbitrary close to each other with high probability and 
therefore the interference may become larger than what is experienced in real network.

Another observation is the extensive use of the Rayleigh faded radio propagation model in the literature for cellular network performance analysis, and hence excluding other types of fading. By relying solely on Rayleigh fading, other important types of fading like shadowing is excluded from the models. We believe that the Rayleigh faded only models suffer from fundamental inaccuracies which should not be ignored. It is well documented that slow fading or shadowing is an important part of radio propagation modelling at least when it comes to macro type of cells where different types of obstacles are likely to be present [11]. In our calculation we demonstrate that themean obtainable bit rate per hertz is a decreasing function of the standard deviation of the shadowing.

In one part of this paper we consider a cellular network where the interfering BSs are uniformly distributed over a given area in the plane, e.g. are outside a cell of circular shape. Thereby we may avoid that interfering BSs may be arbitrary close to the BS a user is connected to.From a modelling perspective it is important that the interfering BSs cannot be arbitrarily close to the BS a user is connected to, however, the possible distance between interfering BSs is not that important since it is the actual distances and not the exact locations that counts. Another approach is to apply more sophisticated stochastic models for BS deployment that have some kind of repulsive properties. We therefore also consider the Ginibre Point Process (GPP) as an option for BS layout [13][14]. In addition we also allows for more realistic fading models by combining fast and slow (shadowing) fading. The fading used is the so-called Suzuki fading model that combines Rayleigh fading and Log-normal slow fading (shadowing) [7]. The aim is to obtain the distribution of the SINR, e.g. the outage performance, but also the bit rate per hertz efficiency for the cellular network which is a very strong performance measure.

The rest of this paper is organised as follows. In section 2 we briefly summarize related work. In section 3 the propagation and fading models used in the analysis is discussed. Then in section 4 the distribution of the SINR is derived for several scenarios ranging from fixed hexagonal cell layout to stochastic location of the interfering BSs. Section 5 describes how the distribution and moments of the bitrate per hertz can be obtained an LTE network, based on the discrete Channel Quality Indicator (CQI) table standardised by 3 GPP. Then in section 6 some numerical examples are discussed and section 7 concludes the paper.

\section{RELATED WORK}

Since the breakthrough of Andrews et al. it in the paper[1],where a PPP is used to describe locations of BSs in cellular network for other cells interference calculations, which also result in surprisingly compact and elegant results for the distribution of SINR and spectrum efficiency, similar analyses have been carried out in several new directions. Interference handling is extremely important for the performance of cellular networks and models that can be used to calculate the effect oncell capacity and on outage probabilities are highly valuable. For instance when deploying LTE networks coverage and capacity estimation will be valuable input for network planners to determine necessary radio resources and secure QoS to the users.

Later works have been extended mainly in the two following directions:

- HetNet type of cellular networks with small cells present, e.g. multiple tiers

- More realistic BS location models than the PPP

The modelling approach in [1] is possible to extend to Het Net type by introducing several tiers. In [15]-[18]different variants of multi tiers analysis are given. The resulting model capture the heterogeneity very well where the BSs in each tier is assumed to be located according to PPPs with different intensities. The main outcome of the modelling is mainly the coverage probability 
under some different assumptions like how users are connected to the different tiers. For most of the cases closed form solutions or/and approximations are found.

Recently other types of stochastic point processes have been proposed to model macro cell layout of BS. One of the promising new approaches is to assume that the BS layout follows a so-called determinantal point process (DPP). The DPP have in contrast to the PPP soft repulsiveness characteristic meaning that the probability of having two BSs to be located very close will be very small. A stationary DPP is characterised by a single covariance function which determines the DPP completely. Different forms of the covariance function give rise to different types of DPPs. In [19] it is demonstrated that DPP very well describes the BS layout found in real deployments. For special case of $\beta$-GPP the distribution of the SINR is found in relative closed forms in [14] for Rayleigh faded transmission. For the general DPP similar expressions are found in [19] but the result is not feasible for calculation in its current form.

\section{MODELLING OF RADIO PROPAGATION AND FADING}

The description of fading of radio signals is an important part of models for spectrum efficiency. Usually the radio propagation is described by a distance dependant part, the path loss, and an additional stochastic fading part that is not distance dependent. The fading, i.e. the stochastic part, is often divided into two distinct components namely slow fading or shadowing and fast fading. Below, we briefly describe the different components and also discuss how it is possible to combine slow and fast fading.

\subsection{PATH Loss MOdel}

One of the most used path loss model for mobile scenarios is the Cost-Hata model, also called the COST 231 model [8]. The path loss $\mathrm{L}$ in $\mathrm{dB}$ is given by:

$$
L=46.3+33.9 * \log (f)-13.82 \log \left(h_{B}\right)-a\left(h_{R}\right)+\left[44.9-6.55 \log \left(h_{B}\right)\right] \log (R)+C
$$

where

$$
a\left(h_{R}\right)=(1.1 \log (f)-0.7) h_{R}-(1.56 \log (f)-0.8) \text { and } C=\left\{\begin{array}{c}
0 d B \text { for mediumcities and suburbanareas } \\
3 d B \text { for metropolitan areas }
\end{array}\right.
$$

and where, $L=$ median path loss in $\mathrm{dB}, f=$ carrier frequency in megahertz, $h_{B}=\mathrm{BS}$ antenna effective height in meters, $R=$ link distance in kilometres, $h_{R}=$ mobile station antenna effective height in meters, $a\left(h_{R}\right)=$ mobile station antenna height correction factor. Its validity ranges are:

- frequency: $1500 \mathrm{MHz}$ to $2000 \mathrm{MHz}$,

- mobile station antenna height: 1 to $10 \mathrm{~m}$,

- BS antenna height: 30 to $200 \mathrm{~m}$ and link distance: 1 to $20 \mathrm{~km}$.

In the numerical examples in this paper we will use: $f=2000 \mathrm{MHz}, h_{B}=30$ meter, $h_{R}=1.5$ meter and $C=0 \mathrm{~dB}$, which then gives: $L=137.744+35.2249 \log (R)$.

\subsection{Shadowing ANd Slow FAding (Log-Normal FAding)}

Log-normal shadowing is the result of the signal being blocked by large objects in the propagation path. These are typically distant objects in the environment such as mountains, hills, or large buildings. The length of time it takes for a moving receiver to pass through the "shadow" of these obstacles brings about the term "slow fading". The statistical model used to describe shadowing is the Log-normal distribution of the mean signal power [9]. Briefly the Log-normal shadowing is obtained by assuming that there is a stochastic part of $L$, say $Y$, added to the expression (1) that is normal distributed (with zero mean) and standard deviation $\sigma_{Y}$ (all given in 
dB). The corresponding variable $X=10^{\frac{\gamma}{10}}=e^{\frac{\ln (0)_{Y} Y}{10} Y}$ is therefore Log-normal distributed with parameters $\mu=0$ and $\sigma=\frac{\sigma_{r} \ln 10}{10}$. The Probability Density Function (PDF) and Cumulative

Complementary Distribution Function (CCDF) of the Log-normal distribution with parameters $\mu=0$ and $\sigma$ are given by:

$$
\operatorname{Ln}_{\sigma}(x)=\frac{1}{\sqrt{2 \pi} \sigma x} e^{-\frac{(\ln x)^{2}}{2 \sigma^{2}}} \text { and } \tilde{L} N_{\sigma}(x)=\frac{1}{2} \operatorname{erfc}\left(\frac{\ln x}{\sigma \sqrt{2}}\right)
$$

where $\operatorname{erfc}(y)=\frac{2}{\sqrt{\pi}} \int_{x=y}^{\infty} e^{-x^{2}} d x$ is the complementary error function.

\subsection{FAST FAding (RAYleigh FAding)}

Fast fading is also called multi-path fading, as a result of multi-path propagation [4]. When multipath signals arrive at a User Equipment (UE), the constructive and destructive phases create rapid variations in signal strength. The worst case fast fading occurs when there is no direct path, which is called Rayleigh fading. Using a one ray model, this small scale distribution simulates the effects of rapid amplitude fluctuations when the receiver travels a distance of a few wavelengths. As the number of reflected rays approaches infinity, the signal levels approaches a Rayleigh distribution, while the corresponding power follows an exponential distribution. In this paper we assume that the fast fading is according to the Rayleigh model.

\subsection{Combining Fast And Slow Fading (Suzuki Distributed Fading)}

Suzuki fading superimposes the Log-normal distribution onto the Rayleigh distribution. This is often used to simulate the effects of a dense urban environment with the average received power level fluctuating slowly due to shadowing effects and the fast fading represents rapid signal fluctuations on top of the shadowing effect [7]. By conditioning on the slow fading then for a Rayleigh faded channel the fast fading component will be exponentially distributed. Hence, the total stochastic variation of the signal power may be taken as the product $S=X_{\ln } X_{e}$ of a Lognormal and an exponential distributed random variable. (By proper scaling we take the mean of the exponential distributed random variable $X_{e}$ to unity.) The resulting distribution also called the Suzuki distribution, and have PDF and CCDF given by the integrals:

$$
f_{S}(x)=\int_{t=0}^{\infty} \frac{1}{t} e^{-\frac{x}{t}} L n_{\sigma}(t) d t \text { and } \widetilde{F}_{S}(x)=\int_{t=0}^{\infty} e^{-\frac{x}{t}} L n_{\sigma}(t) d t
$$

where $L n_{\sigma}(x)=\frac{1}{\sqrt{2 \pi} \sigma x} e^{-\frac{(n x)^{2}}{2 \sigma^{2}}}$ is the Log-normal PDF. Since $\operatorname{Ln}(t)=\frac{1}{t^{2}} \operatorname{Ln} n_{\sigma}\left(\frac{1}{t}\right)$ it is possible to express the integrals above in terms of the Laplace transform of the Log-normal distribution by:

$$
\widetilde{F}_{S}(x)=\hat{f}_{L n_{\sigma}}(x)=\int_{t=0}^{\infty} e^{-t x} L n_{\sigma}(t) d t=\frac{1}{\sqrt{2 \pi} \sigma} \int_{t=0}^{\infty} \frac{e^{-t-\frac{(\ln (t / x))^{2}}{2 \sigma^{2}}}}{t} d t
$$

where $\hat{f}_{L n_{\sigma}}(x)$ is the Laplace transform of the Log-normal distribution. Moreover, the corresponding Laplace transform of $S$ may be written as:

$$
\hat{f}_{S}(x)=E\left[e^{-x S}\right]=\int_{y=0}^{\infty} \frac{y}{y+x} \operatorname{Ln}(y) d y
$$

In a separate appendix we provide some additional results on Suzuki distribution. Especially we show that the CCDF and PDF and the corresponding Laplace transforms may be expressed as contour integrals. These types of integrals are well suited for deriving asymptotic expansions, e.g. 
by applying the saddle point method which turns out to be very accurate.

\section{Outage Probabilities And Distribution Of SiNR}

\subsection{GeNERAL CONSIDERATIONS}

One of the most important parameters determining the cell capacity and obtainable bitrates for users is the Signal to Interference plus Noise Ratio (SINR). We have

$$
\operatorname{SINR}=\frac{P}{N+I}
$$

where $P$ is the received power, $N$ is noise power and $I$ represents the interference from neighbouring cells. In the following we also allow for stochastic location of BSs. So formally we let $\Omega$ be the set of BSs under consideration (which may have stochastic location) and we denote the BS that the user is connected to $B_{0}$ and we denote the set of interfering BSs as $\Gamma=\Omega-B_{0}$. (See Figure 1 below)

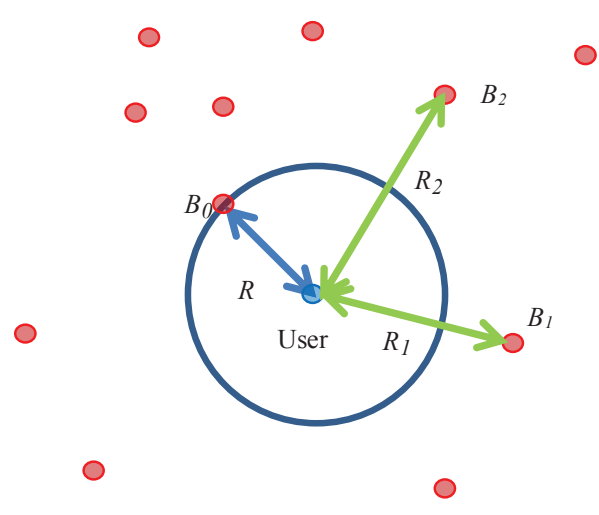

Figure 1. General layout for interference considerations downlink.

Now we assume that $P=a S$ where $S$ represents the stochastic fading (with mean unity) and $a=c \delta R^{-\alpha}$ where $c$ is the transmitted power and $\delta R^{-\alpha}$ represents the path loss where $R$ is the distance to the $\mathrm{BS} B_{0}, \delta$ is an appropriate scaling parameter and $\alpha$ the attenuation factor (a.k.a. the path loss exponent). The interference $I$ is the sum of the received power of all interfering signals from all the other BSs i.e. we take $I=\sum_{i \in \Gamma} a_{i} S_{i}$ where $\Gamma=\Omega-B_{0}$ is the set of interfering BSs and $S_{i}$ represents the stochastic fading component and further $a_{i}=c_{i} \delta R_{i}^{-\alpha}$ where $c_{i}$ is the corresponding transmitted power and $R_{i}$ is the distance to the $i$ ' th interfering $\mathrm{BS} B_{i}$ (where $\left.B_{i} \in \Gamma\right)$. We may there for write $\operatorname{SINR}=\frac{S}{b+\bar{I}} \quad$ where $b=\eta R^{\alpha}$ with $\eta=\frac{N}{c \delta}$ as the noise to signal ratio divided by the scaling factor in the path loss model and the scaled interference $\bar{I}=I / a$ which then may be written as $\bar{I}=\sum_{i \in \Gamma} b_{i} S_{i}$ where $b_{i}=d_{i}\left(R_{i} / R\right)^{-\alpha}$ with $d_{i}=\frac{c_{i}}{c}$.

To find the distribution or outage probabilities of $S I N R$ we first consider the case where the location of the BSs $\Omega$ are known. By conditioning on the interference we find: 
International Journal of Wireless \& Mobile Networks (IJWMN) Vol.9, No.3, June 2017

$$
\widetilde{F}_{\Omega}(x)=\operatorname{Pr}\left(\frac{S}{b+\bar{I}}>x \mid \Omega\right)=\int_{y=0}^{\infty} \operatorname{Pr}(S>(b+y) x \mid \bar{I}=y, \Omega) f_{\bar{I} \mid \Omega}(y) d y=\int_{y=0}^{\infty} \widetilde{F}_{S}\left(\left(y+\eta R^{\alpha}\right) x\right) f_{\bar{I} \mid \Omega}(y) d y
$$

where $\widetilde{F}_{S}(y)=\operatorname{Pr}(S>y)$ is the CCDF of the fading stochastic variable and $f_{\bar{I} \mid \Omega}(y)$ is the PDF of the scaled interference $\bar{I}$ when the locations of the BSs are known. (When there is no confusion we will omit the notation of $\Omega$ for simplicity.) Alternative by writing $\widetilde{F}_{S}(x)$ by its Laplace transform through the inversion formula: $\widetilde{F}_{S}(x)=\frac{1}{2 \pi i} \int_{\gamma_{a}} e^{x z} \hat{F}_{S}(z) d z$ where $\gamma_{a}=\{z=a+i y\}$ is a straight line parallel with imaginary axis with $a \geq 0$, where further $\hat{F}_{S}(z)=\frac{1-\hat{f}_{S}(z)}{z}$ and $\hat{f}_{S}(z)=E\left[e^{-z S}\right]$ is the Laplace transform the fading variable $S$. Plugging in the inversion formula for $\widetilde{F}_{S}(x)$ in (8)and reversing the order of integration gives:

$$
\begin{gathered}
\widetilde{F}_{\Omega}(x)=\frac{1}{2 \pi i} \int_{\gamma} \frac{1-\hat{f}_{S}(z)}{z} H_{\Omega}(-x z) d z \text { with } \\
H_{\Omega}(z)=e^{-z \eta R^{\alpha}} \hat{f}_{\bar{I} \mid \Omega}(z) \text { and }
\end{gathered}
$$

where we also have

$$
\hat{f}_{\bar{I} \mid \Omega}(z)=\int_{y=0}^{\infty} e^{-y z} f_{\bar{I} \mid \Omega}(y) d y=E\left[e^{-z \bar{I}} \mid \Omega\right]=E\left[e^{-z\left(\sum_{i \in \Gamma} b_{i} S_{i}\right)}\right]=\prod_{i \in \Gamma} E\left[e^{-z b_{i} S_{i}}\right]=\prod_{i \in \Gamma} \hat{f}_{S}\left(z d_{i}\left(R_{i} / R\right)^{-\alpha}\right)
$$

which is the Laplace transform of the interference $\bar{I}$ when the locations of all interfering BSs are known. In (9) we must secure that the contour $\gamma_{a}=\{z=a+i y\}$ is chosen so small that also $\hat{f}_{\bar{I} \mid \Omega}(-x z)$ is analytical on the contour, i.e. we may not take a arbitrarily large. Alternative by changing the integration (9) may be written as:

$$
\widetilde{F}_{\Omega}(x)=\frac{1}{2 \pi i} \int_{\gamma^{*}} \frac{\hat{f}_{S}(-z / x)-1}{z} H_{\Omega}(z) d z
$$

where the contour $\gamma_{a^{*}}=\left\{z=a^{*}+i y\right\}$ is in the negative half plane i.e. $a^{*} \leq 0$ but chosen so that also $H_{\Omega}(z)$ is analytically on the line.

For Suzuki faded channels the expression for the outage probability $\widetilde{F}_{\Omega}(x)$ may be heavily simplified. If the fading $S$ is Suzuki distributed then the outage probability is given by:

$$
\widetilde{F}_{\Omega}(x)=\int_{y=0}^{\infty} H_{\Omega}(x y) L n_{\sigma}(y) d y \text { where } H_{\Omega}(z)=e^{-z \eta R^{\alpha}} \prod_{i \in \Gamma} \hat{f}_{S}\left(z d_{i}\left(R_{i} / R\right)^{-\alpha}\right)
$$

To obtain (13) we take (8) as the starting point, and by (5) we find (by changing the order of integration): $\widetilde{F}_{\Omega}(x)=\int_{y=0}^{\infty} \int_{z=0}^{\infty} e^{-\left(y+\eta R^{\alpha}\right) x z} L n_{\sigma}(z) f_{\bar{T} \mid \Omega}(y) d z d y=\int_{z=0}^{\infty} L n_{\sigma}(z) H_{\Omega}(z x) d z$ where

$H_{\Omega}(z)=\int_{y=0}^{\infty} e^{-\left(y+\eta R^{\alpha}\right) z} f_{\bar{I} \mid \Omega}(y) d y=e^{-z \eta R^{\alpha}} E\left[e^{-z \bar{I}} \mid \Omega\right] . \quad$ Now $E\left[e^{-z \bar{I}} \mid \Omega\right]=\prod_{i \in \Gamma} E\left[e^{-z b_{i} S_{i}}\right]=\prod_{i \in \Gamma} \hat{f}_{S}\left(z d_{i}\left(R_{i} / R\right)^{-\alpha}\right) \quad$ since $b_{i}=d_{i}\left(R_{i} / R\right)^{-\alpha}$ and hence we obtain $H_{\Omega}(z)=e^{-z \eta R^{\alpha}} E\left[e^{-z \bar{I}} \mid \Omega\right]=e^{-z \eta R^{\alpha}} \prod_{i \in \Gamma} \hat{f}_{S}\left(z d_{i}\left(R_{i} / R\right)^{-\alpha}\right)$. 
In fact we may also prove (13) by applying (9) and the fact that $\frac{1-\hat{f}_{S}(z)}{z}=\int_{y=0}^{\infty} \frac{1}{y+z} \operatorname{Ln}(y) d y$. By reversing the integration order in the expression for $\widetilde{F}_{\Omega}(x)$ we have $\widetilde{F}_{\Omega}(x)=\int_{y=0}^{\infty} G(x, y) L n_{\sigma}(y) d y$ where $G(x, y)$ is given by the contour integral $G(x, y)=\frac{1}{2 \pi i} \int_{y} \frac{1}{y+z} H_{\Omega}(-x z) d z$. By evaluating the residue at $z=-y$ we then have $G(y, x)=H_{\Omega}(x y)$.

If the slow fading is negligible, i.e. we have a Rayleigh faded channel only, then the outage probability is obtained by taking the limit $\sigma \rightarrow 0$; i.e. we may take $L n_{\sigma}(y)=\delta(1-y)$ and we find:

$$
\widetilde{F}_{\Omega}(x)=H_{\Omega}(x)=e^{-x \eta R^{\alpha}} \prod_{i \in \Gamma} \frac{1}{1+x d_{i}\left(R_{i} / R\right)^{-\alpha}}
$$

To simplify the modelling we shall in the following assume that all the interfering cells are transmitting with the same power i.e. we have $d_{i}=d$ for all $i$, but we allow for cases where the connected BS may transmit with different power than the interfering ones, i.e. we allow for value of $d$ that differs from unity.

\subsection{Fixed HeXagonal GRID}

In this case all the BSs have a fixed location. In the following we give the outage probability for a hexagonal cell layout by taking the interference from the nearest BSs into account. I.e. we neglect the interference from BSs outside the first ring of hexagons. (See Figure 2 below.)

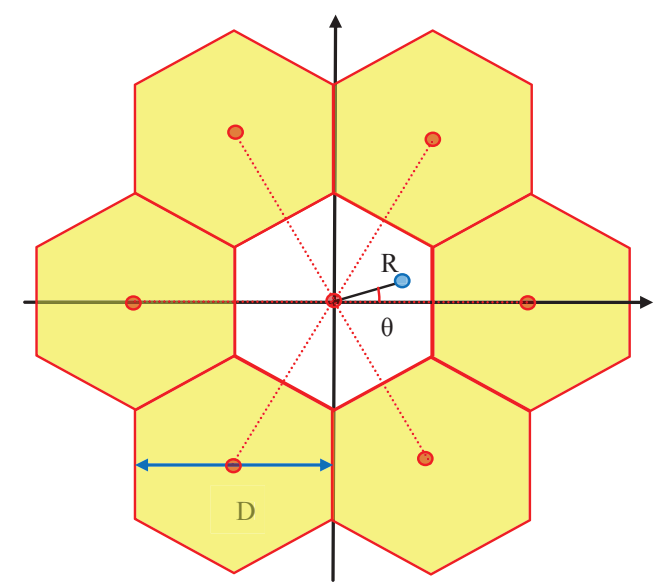

Figure 2. Hexagonal cell layout.

If a user is located in the inner hexagonal by its polar coordinates $(R, \theta)$ and the distance between BSs is taken to be $D$, then the distance to the interfering BSs may be written:

$$
\begin{gathered}
R_{k}=\sqrt{R^{2}+D^{2}-2 R D \cos (k \pi / 3-\theta)} \text { for } k=0,1, . ., 5 \text { and hence we may write } \\
H(z, R, \theta)=e^{-z \eta R^{\alpha}} \hat{f}_{\bar{I}}(z, R, \theta)
\end{gathered}
$$

where $\hat{f}_{\bar{I}}(z, R, \theta)$, the Laplace transform of the interference is given as,

$$
\hat{f}_{\bar{I}}(z, R, \theta)=\prod_{k=0}^{5} \hat{f}_{S}\left(z d\left(\left(1+(D / R)^{2}-2(D / R) \cos (k \pi / 3-\theta) / R\right)^{-\frac{\alpha}{2}}\right)\right.
$$


If we assume that users are uniformly distributed over the cell area, we find the function $H(z)$ by averaging over the cell:

$$
H(z)=\frac{8 \sqrt{3}}{D^{2}} \int_{\theta=0}^{\frac{\pi}{6}} \int_{R=0}^{\frac{D}{2 \cos (\theta)}} R H(z, R, \theta) d R d \theta
$$

Observe that in (17) we may choose the smaller averaging areal than a full hexagonal due to the symmetry.

If a second ring of hexagonal interfering cells is added expression (16) has to be extended by the product of 12 extra Laplace transforms with the appropriate distances while (17) remains the same.

\subsection{STOCHASTIC LOCATION OF BS}

As the number of interfering BSs grows large the computational effort by applying a fixed layout of BS will not be feasible. Another issue is that for a real network there will be large variations in the actual placing of the BSs so a regular grid will not match well with what will be the layout of BSs in a real network. To cater for such variations stochastic models may give a better description of the interference than a regular grid model is able to do.

\subsubsection{UNIFORM DIS TRIBUTION OFBSS}

In the following we assume that the BSs are uniformly distributed over an area $A$ in the plane and that the user is located say at a particular location which we chose as the origin. We assumes that we have totally $n$ BSs that all are uniformly distributed over $A$ and we assume that the user is connected to the nearest base station and that the remaining $n-1$ BSs are interfering the user. We denote $|A|=\int_{A} d A$ the areal of $A$. We shall first find the Laplace transform of the interference given that the distance to the nearest (connected) BS is $R$ and we denote the circle centred at the origin of radius $R$ for $C_{R}$. Hence there are $n-1$ interfering BSs that are uniformly distributed over $A-C_{R}$. And the probability of finding BS $i$ located (by polar coordinates) at $\left(R_{i}, \theta_{i}\right)$ in the surface element $d A_{i}=R_{i} d R_{i} d \theta_{i}$ is $\frac{d A_{i}}{\left|A-C_{R}\right|}$. By (11) we therefore get when un-conditioning on the distances $R_{i}$ of all the interfering BSs:

$$
\hat{f}_{\bar{I} \mid R}(z)=\frac{1}{\left|A-C_{R}\right|^{n-1}} \int_{A-C_{R}} \int_{A-C_{R}} \cdots \int_{A-C_{R}} \prod_{i=1}^{n-1} \hat{f}_{S}\left(z d\left(R_{i} / R\right)^{-\alpha}\right) d A_{1} d A_{2} \cdots d A_{n}=\left(\frac{1}{\left|A-C_{R}\right|} \int_{r \in A-C_{R}} \hat{f}_{S}\left(z d(r / R)^{-\alpha}\right) d A(r)\right)^{n-1}
$$

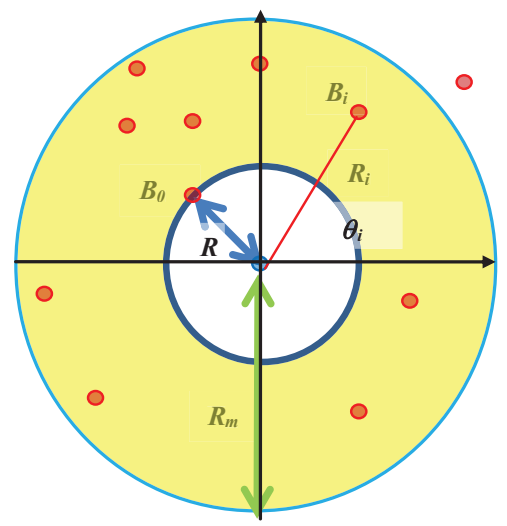

Figure 3. Stochastic cell layout with circular symmetry where the user is located in origin . 
and hence;

$$
H_{n, R}(z)=e^{-z R^{\alpha}}\left(\frac{1}{\left|A-C_{R}\right|} \int_{r \in A-C_{R}} \hat{f}_{S}\left(z d(r / R)^{-\alpha}\right) d A(r)\right)^{n-1}
$$

where the surface integration is taken over the variation of the distance $r$ (over the area $A$ ). The probability that exactly one of the BS is located in a surface element $d A=R d R d \theta$ at distance $R$ and that all the remaining $n-1$ BSs are outside $C_{R}$ is simply $n \frac{d A}{|A|}\left(\frac{\left|A-C_{R}\right|}{|A|}\right)^{n-1}$. Then by integrating over the area $A$ we obtain:

$$
H_{n}(z)=\frac{n}{|A|} \int_{R \in A} e^{-z \eta R^{\alpha}}\left(1-\frac{1}{|A|}\left\{\left|C_{R} \cap A\right|+\int_{r \in A-C_{R}}\left(1-\hat{f}_{S}\left(z d(r / R)^{-\alpha}\right)\right) d A(r)\right\}\right)^{n-1} d A(R)
$$

If we have circular symmetry, e.g. the area $A=C_{R_{m}}$ a circle with radius $R_{m}$, the angular parts of the surface integrals are easily performed giving:

$$
H_{n}(z)=n \int_{y=0}^{1}\left(1-y\left(1+G\left(z, y^{-1}\right)\right)\right)^{n-1} e^{-z y^{\frac{\alpha}{2}}} d y
$$

where now take $\gamma=\eta R_{m}{ }^{\alpha}$ and where $G(z, x)=\int_{u=1}^{x}\left(1-\hat{f}_{S}\left(z d u^{-\frac{\alpha}{2}}\right)\right) d u$. It is possible to write $G(z, x)$ in terms of as a single valued function by just taking:

$$
G(z, x)=G(z)-x G\left(z x^{-\frac{\alpha}{2}}\right) \text { where } G(z)=\int_{u=1}^{\infty}\left(1-\hat{f}_{S}\left(z d u^{-\frac{\alpha}{2}}\right)\right) d u
$$

Now, if we consider a large network where the number of BSs per area is constant $\lambda$, i.e. we have $n=\lfloor\lambda|A|\rfloor$ and $|A| \rightarrow \infty$. Taking the limit, we have: $\frac{n}{|A|} \rightarrow \lambda$ and

$\left(1-\frac{1}{|A|}\left\{\left|C_{R} \cap A\right|+\int_{r \in A-C_{R}}\left(1-\hat{f}_{S}\left(z d(r / R)^{-\alpha}\right)\right) d A(r)\right\}\right)^{\lfloor\lambda|A|\}-1} \rightarrow e^{-\lambda\left(\left.\left|C_{R} \cap A\right|\right|_{r \in A-C_{R}}\left(1-\hat{f}_{S}\left(z d(r / R)^{-\alpha}\right) d d(r)\right)\right.}$ and hence (20) then become:

$$
H(z)=\lambda \int_{R \in A} e^{-z R^{\alpha}} e^{-\lambda\left(\left|C_{R} \cap A\right|+\underset{r e A-C_{R}}{\left.\int\left(1-\hat{f}_{S}\left(z d(r / R)^{-\alpha}\right)\right) d A(r)\right)}\right.} d A(R)
$$

For the case with circular symmetry, e.g. the area $A=C_{R_{m}}$ is a circle with radius $R_{m}$ and $R_{m} \rightarrow \infty$ we obtain:

$$
H(z)=\int_{R=0}^{\infty} 2 \pi R \lambda e^{-z \eta R^{\alpha}-\lambda \pi R^{2}(1+G(z))} d R=\int_{v=0}^{\infty} e^{-q z v^{\alpha / 2}-v(1+G(z))} d v
$$

where $q=\eta(\pi \lambda)^{-\alpha / 2}$. Observe that (23) and (24) is exactly the results as optioned in [1] with the PPP modelling of BSs location. In fact we may easily obtain the results for PPP by the results above. For the PPP the conditional distribution of the of BSs in area $A$ will be uniform, given the 
number of BSs, and the number of BSs will be Poisson distributed with parameter $\lambda|A|$. Hence, for the PPP case we find:

$$
H_{P P P}(z)=e^{-\lambda|| \mid} \sum_{n=0}^{\infty} \frac{(\lambda|A|)^{n}}{n !} H_{n}(z)=\lambda \int_{R \in A} e^{-z \eta R^{\alpha}} e^{-\lambda|| \mid} \sum_{n=1}^{\infty} \frac{1}{(n-1) !}\left(\lambda|A|-\lambda\left\{\left|C_{R} \cap A\right|+\int_{r \in A-C_{R}}\left(1-\hat{f}_{S}\left(z d(r / R)^{-\alpha}\right)\right) d A(r)\right\}\right)^{n-1} d A(R)=H(z)
$$

where $H(z)$ is given by (23). We therefore conclude that for large networks where the BSs are uniformly distributed over the area give exactly the same result as for the corresponding PPP case. This is in fact similar to several other examples where Poisson models are obtained by limiting approaches; e.g. by superposition of thin, independent arrival streams which tend to a Poisson process when the number of streams get large, while the total arrival rate is kept constant.

As pointed out in [1] is it possible to perform the integration of (24) for the cases where $\alpha=2$ and $\alpha=4$. For $\alpha=2$ yields the simple expression:

$$
H(z)=\frac{1}{q z+1+G(z)}
$$

while for $\alpha=4$ the integral may be written in terms of complementary error function:

$$
H(z)=\frac{1}{1+G(z)} T\left(\frac{1+G(z)}{2 \sqrt{q z}}\right) \text { with } T(x)=\sqrt{\pi} x e^{x^{2}} \operatorname{erfc}(x)
$$

By the expressions (21) and (24) the effect of the interference is represented in a very compact manner compared with the expressions for the fixed grid as by (17), (15) and (16) above. To be able to compare the two models the mean numbers of BSs over an area should be equal. Since the area of an hexagon is $\frac{\sqrt{3}}{2} D^{2}$ this corresponds to a BS density of $\lambda=\frac{2}{\sqrt{3} D^{2}}$.

For Suzuki fading it is possible to express the function $G(z)$ more explicitly. We have $G(z)=\int_{y=0}^{\infty} L n_{\sigma}(y)\left(\int_{u=1}^{\infty} \frac{z d u^{-\frac{\alpha}{2}}}{y+z d u^{-\frac{\alpha}{2}}} d u\right) d y$, and the integral in the parenthesis may be expressed by the Hypergeometric function $\frac{2}{\alpha-2} \frac{z d}{y}{ }_{2} F_{1}\left(1,1-\frac{2}{\alpha}, 2\left(1-\frac{1}{\alpha}\right),-\frac{z d}{y}\right)$ and therefore we may express $G(z)$ as:

$$
G(z)=\frac{2}{\alpha-2} \int_{y=0}^{\infty} U(y z) L n_{\sigma}(y) d y \text { with } U(z)=z d_{2} F_{1}\left(1,1-\frac{2}{\alpha}, 2\left(1-\frac{1}{\alpha}\right),-z d\right)
$$

If we assume Rayleigh fading only, the corresponding result is obtained by letting $\sigma \rightarrow 0$; i.e. we may take $L n_{\sigma}(y)=\delta(1-y)$ in (27) and therefore $G(z)=\frac{2}{\alpha-2} U(z)$.

For Log-normal fading distribution we have $G(z)=\int_{y=0}^{\infty} L n_{\sigma}(y)\left(\int_{u=1}^{\infty} 1-e^{-y z d u^{-\frac{\alpha}{2}}} d u\right) d y$. We may write $\int_{u=1}^{\infty}\left(1-e^{-y z d u^{-\frac{\alpha}{2}}}\right) d u=\frac{2}{\alpha} \int_{x=0}^{1}\left(1-e^{-y z d x}\right) x^{-\left(1+\frac{2}{\alpha}\right)} d x$ where last integral will converge for $\alpha>2$. We have $\frac{2}{\alpha} \int_{x=0}^{1}\left(1-e^{-y z d x}\right) x^{-\left(1+\frac{2}{\alpha}\right)} d x=\frac{2}{\alpha}(y z d)^{\frac{2}{\alpha}}\left(\Gamma\left(-\frac{2}{\alpha}, y z d\right)-\Gamma\left(-\frac{2}{\alpha}\right)\right)-1$ where $\Gamma(t)$ and $\Gamma(t, a)$ are the Gamma and the incomplete Gamma function respectively. We may therefore write: 


$$
G(z)=\int_{y=0}^{\infty} V(y z) L n_{\sigma}(y) d y-1 \text { with } V(z)=\frac{2}{\alpha}(z d)^{\frac{2}{\alpha}}\left(\Gamma\left(-\frac{2}{\alpha}, z d\right)-\Gamma\left(-\frac{2}{\alpha}\right)\right)
$$

The case without any fading may be obtained from (28) by taking the limit $\sigma \rightarrow 0$; i.e. we may take $L n_{\sigma}(y)=\delta(1-y)$ and hence $G(z)=V(z)-1$.

Observe that for Log-normal fading we cannot use (13) for calculating the outage distribution, but must rather use the more cumbersome inversion integrals (9) or (12).

\subsubsection{Stochas tic InTerference Models With A Minimum Dis tance B etWeen The CONNECTED AND THE INTERFERING BSS}

If BSs are placed according to a PPP process the BSs will have a finite probability of being very close to each other. In practice there will be a minimum distance between BSs. Also, to be able to compare the results with the fixed grid deployment of BSs we now choose the $\mathrm{BS} B_{0}$ to be at the centre and assume circular symmetry as shown in Figure 4 below, where the interfering BS is located in the area $A$ between circles with radius $R_{\min }$ and $R_{\max }$.

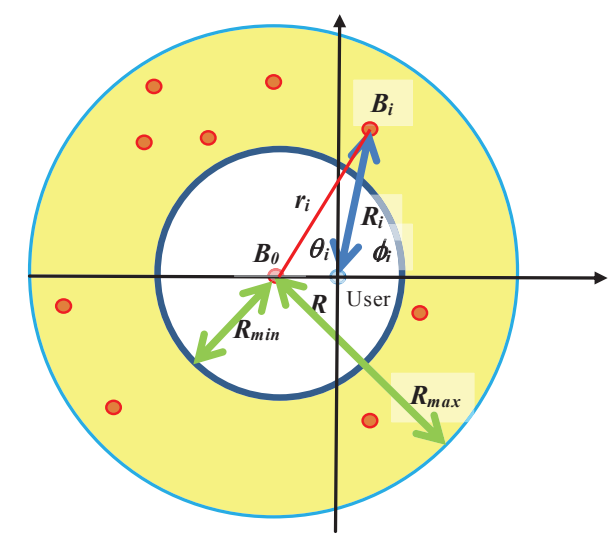

Figure 4. Stochastic location of BSs where we have circular symmetry with the connected BS located at origin.

If there is a total of $n$ interfering BSs which act independently and all of them are located uniformly over the given area, then the Laplace transform of the interference for a particular user located at distance $R$ from the BS the user is connected to may be found by applying the same method as described in section 4.3.1 above and we may apply (18):

$$
\begin{gathered}
\hat{f}_{\bar{I} \mid R}(z)=\left(1-\frac{1}{|A|} \hat{g}_{\bar{I} \mid R}(z)\right)^{n} \text { with } \\
\hat{g}_{\bar{I} \mid R}(z)=\int_{A}\left(1-\hat{f}_{S}\left(z d(v / R)^{-\alpha}\right)\right) d A=2 R^{2} \int_{\phi=0}^{\pi} \int_{r=\left[\left(\phi, R_{\min } / R\right)\right.}^{\Gamma\left(\phi, R_{\max } / R\right)} r\left(1-\hat{f}_{S}\left(z d r^{-\alpha}\right)\right) d r d \phi
\end{gathered}
$$

where $|A|=\pi\left(R_{\max }^{2}-R_{\min }^{2}\right)$ and where the integration limits of $r$ is taken as

$$
\Gamma(\phi, y)=-\cos (\phi)+\sqrt{y^{2}-\sin ^{2}(\phi)} \text { with } y \geq 1
$$

Fortunately, it is possible to perform the integral over the angle $\phi$ in the integral above. To do this we define the following inverse function by: 
International Journal of Wireless \& Mobile Networks (IJWMN) Vol.9, No.3, June 2017

$$
F(y, r)=\left\{\begin{array}{lrl}
0 & \text { for } \quad r<y-1 \\
\arccos \left(\frac{y^{2}-1-r^{2}}{2 r}\right) & \text { for } y-1 \leq r \leq y+1 \\
\pi & \text { for } \quad r>y+1
\end{array}\right.
$$

By reversing the integration and performing the integral over the angle $\phi$ we therefore find:

$$
\hat{g}_{\bar{I} \mid R}(z)=2 R^{2} \int_{r=R_{\min } / R-1}^{R_{\max } / R+1}\left(F\left(R_{\min } / R, r\right)-F\left(R_{\max } / R, r\right)\right) r\left(1-\hat{f}_{S}\left(z d r^{-\alpha}\right)\right) d r
$$

It is possible to write (33) more explicitly by applying (31) and we find:

$$
\begin{gathered}
\hat{g}_{\bar{I} \mid R}(z)=\pi R^{2}\left(Q\left(z,\left(R_{\min } / R\right)\right)-Q\left(z,\left(R_{\max } / R\right)\right)\right) \text { where } \\
Q(z, y)=\frac{2}{\pi} \int_{r=y-1}^{y+1} \arccos \left(\frac{y^{2}-1-r^{2}}{2 r}\right) r\left(1-\hat{f}_{S}\left(z d r^{-\alpha}\right)\right) d r+(y+1)^{2} G\left(z(y+1)^{\alpha}\right)
\end{gathered}
$$

and where $G(z)$ is defined by (19) above. For calculations it may be beneficial to change the integration according to the angle variable $\phi=\arccos \left(\frac{y^{2}-1-r^{2}}{2 r}\right)$, i.e. we take $r=\Gamma(\phi, y)$ where $\Gamma(\phi, y)$ is given by (31) above. With this change of integration variable we obtain:

$$
Q(z, y)=\frac{2}{\pi} \int_{\phi=0}^{\pi} \phi \sin (\phi)\left(\frac{\Gamma(\phi, y)^{2}\left(1-\hat{f}_{S}\left(z d \Gamma(\phi, y)^{-\alpha}\right)\right)}{\sqrt{y^{2}-\sin ^{2}(\phi)}}\right) d \phi+(y+1)^{2} G\left(z(y+1)^{\alpha}\right)
$$

We may also easily analyse the case where the BSs are uniformly distributed on the periphery on a circle of radius $R_{s}$ by letting $P_{\max } \rightarrow R_{\min }=R_{s}$. We therefore find:

$$
\begin{gathered}
\hat{f}_{\bar{I} \mid R}(z)=\left(1-\frac{1}{|L|} \hat{h}_{\bar{I} \mid R}(z)\right)^{n} \text { with } \\
\hat{h}_{\bar{I} \mid R}(z)=-\pi R Q_{y}\left(z,\left(R_{s} / R\right)\right)
\end{gathered}
$$

where $Q_{y}(z, y)=\frac{\partial}{\partial y} Q(z, y)$ is the partial derivative of $Q(z, y)$ and $|L|=2 \pi R_{s}$ is the length of the circumference of the circle. Performing the differentiation gives:

$$
Q_{y}(z, y)=-\frac{4 y}{\pi} \int_{r=y-1}^{y+1} \frac{r\left(1-\hat{f}_{S}\left(z d r^{-\alpha}\right)\right)}{\sqrt{4 r^{2}-\left(y^{2}-1-r^{2}\right)^{2}}} d r
$$

Since (39) is an improper integral for both end points $y-1$ and $y+1$ it will be beneficial also for this integral to introduce the same change of integration variable as above, i.e. according to $r=\Gamma(\phi, y)$ which transforms the integral to:

$$
Q_{y}(z, y)=-\frac{2 y}{\pi} \int_{\phi=0}^{\pi} \frac{\Gamma(\phi, y)\left(1-\hat{f}_{S}\left(z d \Gamma(\phi, y)^{-\alpha}\right)\right)}{\sqrt{y^{2}-\sin ^{2}(\phi)}} d \phi
$$

In the expressions above we have assumed that the numbers of BSs are known, however, over a larger area it will rather be that the number of BSs will be a linear function of the areal of $A$, i.e. 
we have $n=\lfloor\lambda|A|\rfloor$ where $\lambda$ is the density or intensity of BSs per unit area as for the Poisson case above, i.e. we have $\hat{f}_{\bar{I} \mid R}(z)=\left(1-\frac{1}{|A|} \hat{g}_{\bar{I} \mid R}(z)\right)^{\lfloor\lambda|A|\rfloor}$. As the network grows large this will approach an exponential expression:

$$
\hat{f}_{\bar{I} \mid R}(z)=e^{-\lambda \hat{g}_{|| R}(z)} \text { when }|A| \rightarrow \infty
$$

where $\hat{g}_{\bar{I} \mid R}(z)=\int_{A}\left(1-\hat{f}_{S}\left(z d(v / R)^{-\alpha}\right)\right) d A$ and is given above by (34) above for case where $A$ is the area limited between two concentric circles. In the limit when $R_{\max } \rightarrow \infty$ we then find, since $Q\left(z,\left(R_{\max } / R\right)\right)$ tends to zero, the following expressions for $\hat{g}_{\bar{I} \mid R}(z)$ :

$$
\hat{g}_{\bar{I} \mid R}(z)=\pi R^{2} Q\left(z,\left(R_{\min } / R\right)\right)
$$

where $Q(z, y)$ is given either by (35) or (36) above.

Finally the same method will also apply for the case where the BSs are randomly distributed over the periphery of a circle. By taking $n=\left\lfloor\lambda^{*}|L|\right\rfloor$ in (37); then for large $|L|$ we have:

$$
\hat{f}_{\bar{I} \mid R}(z)=e^{-\lambda^{n} \hat{h}_{i \mid R}(z)} \text { where }
$$

$\hat{h}_{\bar{I} \mid R}(z)$ is given by (38) above and where $\lambda^{*}$ now is the BS intensity per length rather than per area.

For all the models above we then have the Laplace transform of the sum of the noise to signal ratio and the scaled interference for a user located at distance $R$ from the transmitting BS on the form:

$$
H_{R}(z)=e^{-z \eta R^{\alpha}} \hat{f}_{\overline{\mid} \mid R}(z)
$$

where the Laplace transform of the interference $\hat{f}_{\bar{I} \mid R}(z)$ is given by either (29), (37), (41) or (43)above.

Finally, if we consider a circular cell with radius $R_{\text {cell }}$ where $R_{\text {cell }} \leq R_{\min }$ and assume that a typical user is uniformly located in the cell, we find by averaging over the cell area:

$$
H(z)=\frac{2}{R_{\text {cell }}^{2}} \int_{R=0}^{R_{\text {cell }}} R H_{R}(z) d R
$$

The circular cell assumption is fundamentally different from the PPP assumption since we restrict the distances between the connected and the interfering BSs. This is not the case for the PPP model where the distance between the connected and the interfering BS may be small with relative high probability. We therefore expect that the PPP modelling will give higher inference than the models in section 4.3.2 and therefore give poorer performance.

\subsubsection{Bss Dis tributed ACCording To A Ginibre Point Proces S}

Recently the Ginibre Point Process (GPP) has gain large attention for describing deployment of BSs in cellular networks. The GPP is a special case of Determinantal Point Process (DPP) which 
has repulsion characterises imolying that two BSs will be close with small probability, while for a PPP there is a tendency to clumping of points that will not been seen in real deployment. It is possible to generalize a GPP to a so called $\beta$-GPP which is a thinned and re-scaled GPP. The thinning is done by deleting the points in the original GPP with probability $\beta$. Rescaling is done to maintain the same intensity as in the standard GPP. The $\beta$-GPP thus extends the classical GPP to point processes that covers a rather broad range of possible BS layouts ranging from PPP when $\beta \rightarrow 0$ to standard GPP when $\beta=1,[12]$.

The derivation below is in line with [13] but the similar result is also derived in [14]. We let $\Omega=\{\Phi, T\}$ where $\Phi$ is where be a point process in the plane with points $X_{i}, i \in N$ where the ordering is arbitrary and $T$ is defined a set of independent indicator variable $T_{i}, i \in N$ with $P\left(T_{i}=0\right)=1-\beta$ and $P\left(T_{i}=1\right)=\beta$. Hence the $\beta$-tinned version of $\Phi$ is the set of points $X_{i}$ which have $T_{i}=1$ and only this points represent the location of a real BSs. We also denote $\left.N_{T}=\left\{i \in N \mid T_{i}=1\right\}\right\}$. Then conditioned on $\Omega=\{\Phi, T\}$ the Laplace transform $H_{\Omega}(z)$ as given by (10) and (11) will take the form:

$$
H_{\Omega}(z)=e^{-z \eta\left(R_{B_{0}}\right)^{\alpha}} \prod_{i \in N_{T} \backslash\left\{B_{0}\right\}} \hat{f}_{S}\left(z d\left(R_{i} / R_{B_{0}}\right)^{-\alpha}\right)
$$

where $R_{i}=\left|X_{i}\right|$ is the distance from the typical user to the BS $i \in N_{T}$ and $B_{0} \in N_{T}$ is the index of the BS where the typical users is associated and we also must have $R_{i}>R_{B_{0}}$ for all $i \in N_{T} \backslash\left\{B_{0}\right\}$.

Summing over all the possibility of $B_{0}=j$ and taking into account that this represents a BS with probability $P\left(T_{i}=1\right)=\beta$ and then we may find $H(z)$ by talking expectations over the random variables $R_{i}$ and the indicator variable $T_{i}$ (this makes it possible to take the product over all $N$ in (47) since $\hat{f}_{S}\left(z d T_{i}\left(R_{i} / R_{j}\right)^{-\alpha}\right)=\hat{f}_{S}(0)=1$ when $\left.T_{i}=0\right)$ :

$$
H(z)=\beta \sum_{j=1}^{\infty} E\left[e^{-z \eta\left(R_{j}\right)^{\alpha}} \prod_{i \in N\{j\}} \hat{f}_{S}\left(z d T_{i}\left(R_{i} / R_{j}\right)^{-\alpha}\right)\right]=\beta \sum_{j=1}^{\infty} E\left[e^{-z \eta\left(R_{j}\right)^{\alpha}} \prod_{i \in N\{\langle j\}}\left(1-\beta+\beta \hat{f}_{S}\left(z d\left(R_{i} / R_{j}\right)^{-\alpha}\right) \mathbf{1}_{R_{j}<R_{i}}\right)\right]
$$

We now use the highly special result for the Ginibre process (see e.g. [13] or [14]) which states that the ensemble $\left\{R_{i}{ }^{2}\right\}$ for $i \in N$ all are independent and $R_{i}{ }^{2}$ follows an Erlang' $i$ distribution with parameter $\gamma=\frac{\pi \lambda}{\beta}$. $\beta$ is here includes to scale up the standard Ginibre process so that all the $\beta$ GPP will have the same intensity $\lambda$. Hence, $R_{i}{ }^{2}$ will have the density function

$$
f_{R_{i}^{2}}(x)=\frac{\gamma(2 x)^{i-1} e^{-\gamma x}}{(i-1) !}
$$

and by inserting in we then have:

$$
H(z)=\beta \sum_{j=1}^{\infty} \int_{u=0}^{\infty} \frac{\gamma(\gamma u)^{j-1} e^{-\gamma u}}{(j-1) !} e^{-z \eta u^{\alpha / 2}} \prod_{i \in N\{j\}\}}\left(1-\beta+\beta \int_{x=u}^{\infty} \frac{\gamma(\gamma x)^{i-1} e^{-\gamma x}}{(i-1) !} \hat{f}_{S}\left(z d(x / u)^{-\alpha / 2}\right) d x\right) d u \text {. After some }
$$

manipulations we then find $H(z)$ can be written:

$$
H(z)=\beta \int_{u=0}^{\infty} e^{-u-q z(\beta u)^{\alpha / 2}} P(z, u) S(z, u) d u
$$

where $q=\eta(\pi \lambda)^{-\alpha / 2}$ and 


$$
P(z, u)=\prod_{i=0}^{\infty}(1-\beta+\beta I(i, z, u)) \text { and } S(z, u)=\sum_{j=0}^{\infty} \frac{u^{j}}{j !}(1-\beta+\beta I(j, z, u))^{-1}
$$

and the integral

$$
I(j, z, u)=\frac{1}{j !} \int_{x=u}^{\infty} x^{j} e^{-x} \hat{f}_{S}\left(z d(x / u)^{-\alpha / 2}\right) d x
$$

For numerical calculations and it will be beneficial to rewrite the product and sum in (50) above by taking $I(j, z, u)=1-J(j, z, u)$. This then gives $P(z, u)=\prod_{i=0}^{\infty}(1-\beta J(i, z, u)) \quad$ and $S(z, u)=\sum_{j=0}^{\infty} \frac{u^{j}}{j !}(1-\beta J(j, z, u))^{-1}$ andby some manipulations we find

$$
J(j, z, u)=e^{-u} R_{j}(u)+\frac{u^{j+1}}{j !} \int_{x=1}^{\infty} x^{j} e^{-x u}\left(1-\hat{f}_{S}\left(z d x^{-\alpha / 2}\right)\right) d x
$$

where $R_{j}(u)=\sum_{l=j+1}^{\infty} \frac{1}{l} u^{l}$ is the $j$ 'th rest in the "truncated" exponential series. By for instance summing $J(j, z, u)$ over all values of $j$ we obtain a linear relation in the variable $u$. We find

$$
\sum_{j=0}^{\infty} J(j, z, u)=u+u \int_{x=1}^{\infty}\left(1-\hat{f}_{S}\left(z d x^{-\alpha / 2}\right)\right) d x
$$

since $\sum_{j=0}^{\infty} R_{j}(u)=\sum_{j=0}^{\infty} \sum_{l=j+1}^{\infty} \frac{1}{l !} u^{l}=\sum_{l=1}^{\infty} \frac{1}{l !} u^{l} \sum_{j=0}^{l-1} 1=\sum_{l=1}^{\infty} \frac{1}{(l-1) !} u^{l}=u e^{u}$ and $\sum_{j=0}^{\infty} \frac{u^{j+1}}{j !} x^{j}=u e^{x u}$.The relation (53) may for instance be used to find the limit $\beta \rightarrow 0$ of $H(z)$. We have $H(z)=\int_{u=0}^{\infty} e^{-u / \beta-q z u^{\alpha / 2}} P(z, u / \beta) S(z, u / \beta) d u . \quad$ When $\quad \beta \rightarrow 0 \quad$ then $e^{-u / \beta} S(z, u / \beta)=e^{-u / \beta} \sum_{j=0}^{\infty} \frac{(u / \beta)^{j}}{j !}(1-\beta J(j, z, u / \beta))^{-1} \rightarrow e^{-u / \beta} \sum_{j=0}^{\infty} \frac{(u / \beta)^{j}}{j !}=1 \quad$ and $\quad$ hence $H(z) \rightarrow \int_{u=0}^{\infty} e^{-q z u^{/ / 2}} P(z, u / \beta) d u$. But the product may be written as a sum of logarithms by $P(z, u / \beta)=e^{\sum_{i=0}^{\infty} \ln (1-\beta J(i, z, u / \beta)}$ and by expanding the logarithms to first order, since $\beta J(i, z, u / \beta)$ will be small for large $i$, we find $\sum_{i=0}^{\infty} \ln \left(1-\beta J(i, z, u / \beta) \approx-\beta \sum_{i=0}^{\infty} J(i, z, u / \beta)=-u-u \int_{x=1}^{\infty}\left(1-\hat{f}_{S}\left(z d x^{-\alpha / 2}\right)\right) d x=-u(1+G(z))\right.$ by $(53)$ where $G(z)$ is defined by (22). And hence $H(z) \rightarrow \int_{u=0}^{\infty} e^{-q z u^{\alpha / 2}-u(1+G(z))} d u$ which is exactly (24) for the PPP. When calculating the product $P(z, u)=\prod_{i=0}^{\infty}(1-\beta J(i, z, u))$ it will be beneficial to factor out the "PPP" part by using (53) since we may write: 


$$
P(z, u)=e^{-u \beta(1+G(z))} e^{T(z, u)} \text { with } T(z, u)=\sum_{j=0}^{\infty}(\ln (1-\beta J(j, z, u))+\beta J(j, z, u))
$$

and similar we also define:

$$
W(z, u)=1+e^{-u} \beta \sum_{j=0}^{\infty} \frac{u^{j}}{j !} \frac{J(j, z, u)}{1-\beta J(j, z, u)}=e^{-u} S(z, u)
$$

Hence, we may therefore write:

$$
H(z)=\int_{u=0}^{\infty} e^{-q z u^{\alpha / 2}-u(1+G(z))} U(z, u / \beta) d u \text { with } U(z, u)=W(z, u) e^{T(z, u)}
$$

The function $U(z, u / \beta)$ therefore represents the deviation from the corresponding PPP model.

Compared to (24) the result for $\beta$-GPP above is much more time consuming to calculate since it requires calculation of both infinite sums as well as numerical integrations. By the rewriting done by (54) and (55) we speed up the convergence of the sums above since $\ln (1-\beta J(j, z, u))+\beta J(j, z, u) \approx-(\beta J(j, z, u))^{2}$ and $\frac{u^{j}}{j !} \frac{\beta J(j, z, u)}{1-\beta J(j, z, u)} \approx \frac{u^{j}}{j !} \beta J(j, z, u)$ due to the fact that $J(j, z, u) \rightarrow 0$ when $j \rightarrow \infty$.

\subsubsection{Formulas For SinR Dis tribution (OUtage Probabilities)}

We end this chapter by summarizing the methods to obtain the SINR distribution. For all the scenarios of BSs locations described above in 4.2 and 4.3 we can express the outage probabilities by some of the following expressions:

- for Rayleigh faded channels:

$$
\widetilde{F}(x)=H(x)
$$

- and Suzuki faded channels by the integral [15]:

$$
\widetilde{F}(x)=\int_{y=0}^{\infty} H(x y) L n_{\sigma}(y) d y=\frac{1}{x} \int_{y=0}^{\infty} H(y) L n_{\sigma}(y / x) d y
$$

- and finally for general fading distribution $S$ (with Lace transform $\hat{f}_{S}(z)$ ) the outage is given by the contour integrals (9) or (12):

$$
\widetilde{F}(x)=\frac{1}{2 \pi i} \int_{\gamma} \frac{1-\hat{f}_{S}(z)}{z} H(-x z) d z=\frac{1}{2 \pi i} \int_{\gamma^{*}} \frac{\hat{f}_{S}(-z / x)-1}{z} H(z) d z
$$

In All These Cases Represent The Laplace Transform Of The "Scaled" Interference And Noise Power And Is Given As Either (17), (21), (24), (45) Or (56). In Appendix- III We Discuss Some (Numerical) Methods For Evaluation Of The Integral (58) For Suzuki Fading. )

\section{Capacity Considerations Based On LTE-CQI Values}

In this section we analyse the bit rate that is possible to obtain of a channel of a certain bandwidth. The analysis is based on the assumption that it is possible to specify a functional 
relation between the SINR and the corresponding bit rate. For LTE part of this relation is given by the 15 standardized CQI values in Table 1 below [2]. To fully specify this relation the mapping between SINR values to different CQI indexes also have to be specified. Often a linear relation between CQI indexes and SINR in $[\mathrm{dB}]$ is assumed, but this may be vendor specific. The absolute upper bound on the bit rate-SINR relation is of course the Shannon formula [10].

\subsection{Obtainable Bitrate Per Symbol Rate as Function Of SinR}

The aim is to express the bit rate as a function of SINR for a user in an LTE cell. For LTE the obtainable spectrum efficiency will depend on the radio signal quality (both for up-and downlink). An indication of the actual radio signal quality is signalled over the radio interface by the so-called CQI index, which is in the range 1 to 15 . Based on the CQI value the coding rate is determined on basis of the modulation QPSK, 16QAM, 64QAM, and the amount of redundancy included. The modulation and the Forward Error Correction (FEC) rate is chosen based on the CQI value as shown in Table 1 [2]. For analytical modelling the actual CQI measurement procedures are difficult to incorporate into the analysis due to the time lag, i.e. the signalled CQI is based on measurements taken in earlier TTIs (Transmission Time Interval). To simplify the analyses, we assume that this time lag is set to zero and that the CQI is given as a function of the momentary SINR, i.e. CQI = CQI(SINR). This approximation is justified if the time variation in SINR is significantly slower than the length of a TTI interval. Hence, by applying the CQI table found in [2] we get the spectrum efficiency as function of the SINR as the step function:

$$
B=f c_{j} \quad \text { for } \operatorname{SINR} \in\left[g_{j}, g_{j+1}\right) \text { for } j=0,1, \ldots, 15
$$

where $f$ is the bandwidth of the channel, $c_{j}$ is the efficiency for CQI equal $j$ (as given by Table 1) and $\left(g_{j}, g_{j+1}\right)$ are the corresponding intervals of SINR values. (We also take $c_{0}=0, g_{0}=0$ and $g_{16}=\infty$.)

Table 1 CQI table

\begin{tabular}{|c|c|c|c|}
\hline CQI index & modulation & code rate x 1024 & efficiency \\
\hline \hline 0 & \multicolumn{3}{|c|}{ out of range } \\
\hline 1 & QPSK & 78 & 0.1523 \\
\hline 2 & QPSK & 120 & 0.2344 \\
\hline 3 & QPSK & 193 & 0.3770 \\
\hline 4 & QPSK & 308 & 0.6016 \\
\hline 5 & QPSK & 449 & 0.8770 \\
\hline 6 & QPSK & 602 & 1.1758 \\
\hline \hline 7 & 16QAM & 378 & 1.4766 \\
\hline 8 & 16QAM & 490 & 1.9141 \\
\hline 9 & 16QAM & 616 & 2.4063 \\
\hline \hline 10 & 64QAM & 466 & 2.7305 \\
\hline 11 & 64QAM & 567 & 3.3223 \\
\hline 12 & 64QAM & 666 & 3.9023 \\
\hline 13 & 64QAM & 772 & 4.5234 \\
\hline 14 & 64QAM & 873 & 5.1152 \\
\hline 15 & 64QAM & 948 & 5.5547 \\
\hline
\end{tabular}

To fully describe the bit rate function above we also have to specify the intervals $\left(g_{j}, g_{j+1}\right)$. Several simulation studies e.g. [3] suggest that there is a linear relation between a CQI index and the actual SINR limits in $[\mathrm{dB}]$. With this assumption we have $\operatorname{SINR}_{j}[d B]=10 \log _{10} g_{j}=a j+b$ or $g_{j}=10^{\frac{a j+b}{10}}$ for some constants $a$ and $b$. It is also argued that the actual range of the SINR limits in $[\mathrm{dB}]$ is determined by the following (end point) observations: $\operatorname{SINR}[\mathrm{dB}]=-6$ corresponds to 
$\mathrm{CQI}=1$, while $\mathrm{SINR}[\mathrm{dB}]=20$ corresponds to $\mathrm{CQI}=15$. Hence with this assumption we then have $-6=a+b$ and $20=15 a+b$ or $a=13 / 7$ and $b=-55 / 7$.

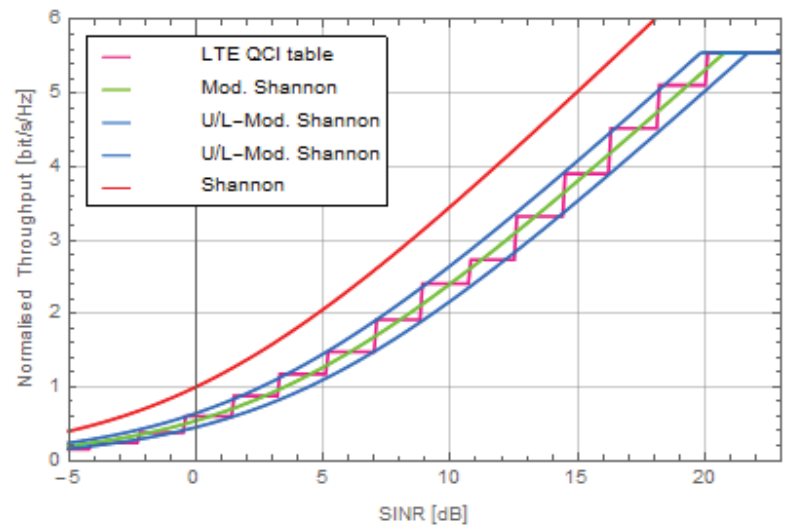

Figure 5. Normalized throughput as function of the SINR based on: 1.-CQI table, 2.-Shannon and 3.Modified Shannon.

For extensive analytical modelling the step-based bandwidth function is somehow cumbersome to apply. An absolute upper bound yields the Shannon formula [10];

$$
B=f \log _{2}(1+\operatorname{SINR})
$$

However, it is well known that the Shannon upper bound is too optimistic. First of all the bandwidth function should never exceed the highest possible rate $c_{15}=5.5547$. We therefore suggest a downscaling and truncating the Shannon formula by taking:

$$
B=d \operatorname{Min}[T, \ln (1+\gamma \operatorname{SINR})]
$$

with $d=f \frac{C}{\ln 2}$ and $T=\frac{c_{15} \ln 2}{C}$ where $C$ is the downscaling constant (relative to the Shannon formula) and $\gamma$ is a constant less than unity. By choosing $C$ and $\gamma$ that minimise the square distances between the standardised CQI values in Table 1above and the truncated Shannon formula (62) we find $C=0.9449$ and $\gamma=0.4852$. (Upper and lower estimates of the CQI based zigzagging bit rate function is obtained by taking $\gamma_{u}=\gamma 10^{a / 20}=0.6008$ and $\gamma_{l}=\gamma 10^{-a / 20}=0.3918$ ). We observe that a downscaling of the Shannon limit is very much in line with the corresponding bitrates obtained by the CQI table as shown in Table land hence we believe that (62) yields a quite accurate approximation. In fact the approximated CQI values $c_{j}^{a p p}$, based on the truncated Shannon formula, follow the similar logarithmic behaviour:

$$
c_{j}^{a p p}=C \log _{2}\left(1+\alpha \beta^{j}\right)
$$

where we now have $\alpha=\gamma 10^{a / 20^{+1 / 10}}=0.0984$ and $\beta=10^{\% / 10}=1.5336$. As seen from Figure 6 this approximation is very much in line with the values given by the CQI table. 


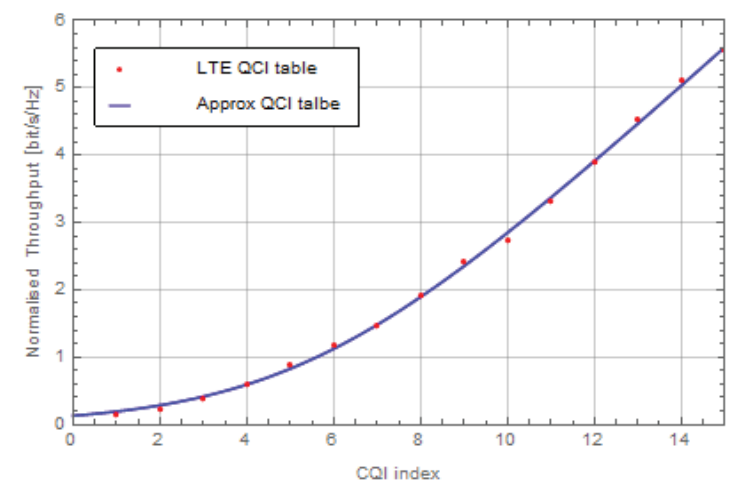

Figure 6. Normalised throughput as function of CQI index based on CQI table and on approximated CQI values.

\subsection{Distribution Of The ObTainable Bitrate For A Channel}

If the distribution of the SINR is known the corresponding distribution of the obtainable bitrate $B$ of a transmission channel, may be found by the distribution of SINR and the functional relation between them. For LTE the functional relation is described in details in section 5.1. Alternatively, this relation may be taken as the Shannon formula which then gives the upper bound of the obtainable bit rate. In section 5.1 we also have determined a truncated version of Shannon formula that approximates the standardized bitrates quite well. Below we express the distribution of the possible obtainable bit rate as function of SINR and the CCDF of SINR given by $\widetilde{F}(x)=\operatorname{Pr}(\operatorname{SINR}>x)$.

First we consider the case where the functional relation between SINR and bit rate $B$ is the step function given by CQI rates inTable 1, i.e. on the discrete form (60) above. The CCDF of the bit rate $B=P(B \leq y)$ is then given as the following step function:

$$
B(y)=1-\widetilde{F}\left(g_{j+1}\right) \quad \text { for } \quad y \in\left(f c_{j}, f c_{j+1}\right] \text { for } j=0,1, \ldots, 15
$$

Hence, we also find the corresponding $k$ 'th moment of the obtainable bitrate as the (finite) sum:

$$
m_{k}=E\left[B^{k}\right]=f^{k} \sum_{j=1}^{15}\left(c_{j}^{k}-c_{j-1}^{k}\right) \widetilde{F}\left(g_{j}\right)
$$

Rather than applying the discrete modelling approach above, we may prefer to apply the continuous counterpart defined by relation (62). With this assumption we find:

$$
B(y)= \begin{cases}1-\widetilde{F}\left(\gamma^{-1}\left(e^{y / d}-1\right)\right) & \text { for } y / d<T \\ 1 & \text { for } y / d \geq T\end{cases}
$$

and based on (66) we may write the $k$ 'th moment of the bit rate as:

$$
m_{k}=E\left[B^{k}\right]=d^{k} \int_{y=0}^{\gamma^{-1}\left(e^{T}-1\right)}(\ln (1+\gamma y))^{k} f(y) d y+d^{k} T^{k} \widetilde{F}\left(\gamma^{-1}\left(e^{T}-1\right)\right)
$$

where $f(y)$ is the PDF of the SINR (given as $f(y)=-\widetilde{F}^{\prime}(y)$ ). Alternative by integrating by parts the $k$ 'th moment may also be written in terms of the CCDF as: 


$$
m_{k}=k \gamma d^{k} \int_{y=0}^{\gamma^{-1}\left(e^{T}-1\right)} \frac{(\ln (1+\gamma y))^{k-1}}{1+\gamma y} \widetilde{F}(y) d y
$$

Normally, the moments without any truncation, is found from the expression above by taking the limit $T \rightarrow \infty$. By defining $\eta=\gamma^{-1}\left(e^{T}-1\right)$ then $m_{k}=d^{k}\left(\int_{y=0}^{\eta}(\ln (1+\gamma y))^{k} f(y) d y+\left(\ln (1+\gamma \eta)^{k} \widetilde{F}(\eta)\right)\right.$

. If the limit $\eta \rightarrow \infty$ exists for (67) then we have: $\left(\ln (1+\gamma \eta)^{k}\right) \widetilde{F}(\eta) \leq \int_{y=\eta}^{\infty}(\ln (1+\gamma y))^{k} f(y) d y \rightarrow 0$ by definition; and therefore:

$$
m_{k}=d^{k} \int_{y=0}^{\infty}(\ln (1+\gamma y))^{k} f(y) d y=k \gamma d^{k} \int_{y=0}^{\infty} \frac{(\ln (1+y y))^{k-1}}{1+\gamma y} \widetilde{F}(y) d y
$$

\section{NUMERICAL EXAMPLES AND DISCUSSION}

\subsection{Scenarios ANd Parameter ChOICES For CoMParison OF The Models}

When comparing the different interference models/scenarios described in the sections 4.2 and 4.3 we must be clear about the actual differences between them so that the comparison is "fair". We take the hexagonal layout as the basis for the comparison. In the examples we have calculations for both 7 (Hexagonal 7- BS) and 19 (Hexagonal 19-BS) BSs where the first represents the cases shown in Figure 2 with a BS surrounded by 6 (interfering) neighbouring BSs and the second represents the case with the second ring with additional 12 (interfering) BSs. When comparing hexagonal layout with circular cell models we choose to require that areas are equal, i.e. the area of a circular cell equals that of a hexagonal cell. Also when comparing models with PPP we take the intensity so that the (mean) number of BSs coincide with that of the hexagonal grid i.e. $\lambda A_{\text {hex }}=1$ where $A_{\text {hex }}=(\sqrt{3} / 2) D^{2}$ is the area of a hexagon with (shortest) diameter $D$ as shown in Figure 2. We have considered the following interference scenarios:

1. The cells and BSs are located as hexagonal either with 7 or 19 BSs.

2. BSs are distributed according to a PPP in the plane.

3. BSs are uniformly distributed over the considered area, either with 7 BS over a circular area equal to 7 hexagonal cells, or with $19 \mathrm{BS}$ over a circular area equal to 19 hexagonal cells.

4. The UE is randomly located in a circular cell (with the same area as the hexagonal cells). The interfering BS is located uniformly outside the cell with some different options:

a. Semi-infinite with rate that matches the hexagonal grid.

b. BSs are located outside the cell but inside a limiting circle and where the number of BSs matches the hexagonal cases, i.e. 6 or 18 and the corresponding network area equals that of 7 or 19 hexagonal cells.

c. 6 BSs are located at the periphery of a circle with radius equal to the distance of the interfering BSs from the 7 hexagonal cell case.

In the examples described below we choose the Cost-Hata path loss model with the parameters described in section 3.1. We further assume a transmission power of $2.0 \mathrm{~kW}$ and a noise power of $8.283 \times 10^{-14} \mathrm{~W}$. This gives necessary parameters for the numerical calculations: $\eta=0.0024638$ and $\alpha=3.52249$ All the distances are measured in $\mathrm{km}$. The diameter of the (hexagonal cells) are taken to be $D=2.0 \mathrm{~km}$. 


\subsection{The DeViated HeXagonal Model}

We shall also consider the following planning scenario for deploying BSs in a cellular network: An operator wants to deploy a network of macro BSs in a particular area. As a start, the operator takes a map of the area and marks the initial tentative BS locations according to a regular hexagonal pattern. Then each ideal BS site is inspected and typically moved from its initial location to a nearby location where it is more practical to put it. A simple statistical model for this process is that each $\mathrm{BS}$ is placed randomly in a disc of a certain radius centred at the initial "ideal" regular hexagonal BS locations, as illustrated in Figure 7.

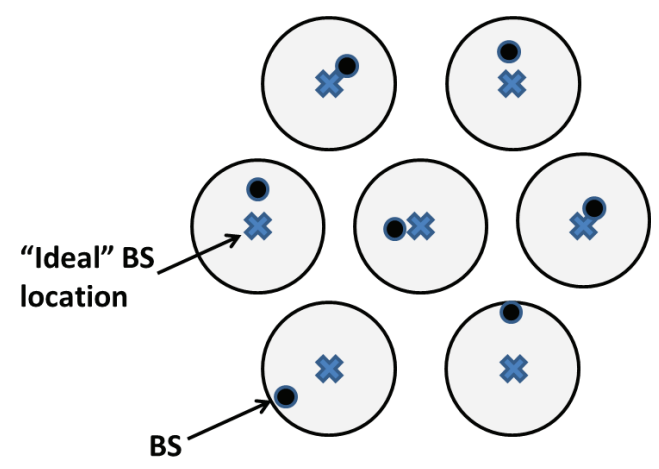

Figure 7.The deviated hexagonal model for selecting BS locations. Crosses indicate the "ideal" BS locations and the black dots the actual selected BS locations .

The location of each BS is assumed to have a uniform probability distribution over the area covered by each disc and the cells are defined by the Voronoi tessellation based on the BS locations. This model is then more realistic than the regular hexagonal models. The BSs are placed more randomly and when the radius of the disc is large the generated BS locations are expected to look very similar to what is obtained with the PPP model. The performances measures obtained with the deviated hexagonal model are therefore expected to lie between the performances found with the regular hexagonal models and the PPP model. For small disc sizes, the performance should be similar to what it is for the regular hexagonal model. But as the disc sizes are increased, the performance is expected to approach the PPP performance.

The main weakness of the PPP model is that BSs can be placed arbitrarily close together, whereas the minimum inter-BS distance can be controlled in the deviated hexagonal model. Hence, the accuracy of the PPP model can be tested by comparing its performances with those obtained with a deviated hexagonal model with large disc sizes, where the disc sizes are chosen to guarantee a certain minimum BS separation.

A simulation study was performed to compare the different analytical models with the deviated hexagonal model, and also to justify the numerical calculations. Both the numerical calculations and simulation was performed by applying the input parameters in 0above and we conclude excellent coincidence between the calculated and the simulated SINR distributions.

\subsection{Probability Of Coverage}

Below we give the coverage probability as a function of the threshold (in $\mathrm{dB}$ ), which is also the CCDF of the SINR, for the various scenarios described above. Four different values for the standard deviation of the shadowing have been chosen in the examples below. These are $0,3,6$, and $9 \mathrm{~dB}$ for the different plots.

In top left of Figure 8-Figure 10 we show the results without any shadowing. This case gives the best performance of all the cases considered. This corresponds to the results presented in [1] 
where the modelling is based on the assumption of having Rayleigh the faded channels. When comparing the effect of shadowing we observe that the shape differs for the different value of the standard deviation. We observe that the coverage performance clearly decrease as function of increase of the shadowing. Also the shape of the coverage curves change depending of the shadowing, while for standard deviation of 0 and $3 \mathrm{~dB}$ have the typical s-shape, the plots with 9 $\mathrm{dB}$ shows a more linear shape without any clear inflection points.

In Figure 8 we compare the different types of cell structures from PPP, uniform location over circular area and the traditional hexagonal layout with 7 and 19 BSs and we observe the rather big difference between the hexagonal cases and the PPP model. The uniform distributed scenarios with 7 and 19 BSs (and with area equal to the corresponding hexagonal cases) give better performance than PPP but are well below the hexagonal cases. The model with circular serving cell and where interfering BSs uniformly distributed outside the serving cell (lilac curves) gives coverage performance which is much in the middle between the hexagonal cases and the PPP model. It is likely that an actual effect of the interference is somehow between these two scenarios and therefore the uniform outside circular cell model in 4.3 .2 actually will give good accordance with real deployments.

One of the main drawbacks with the PPP modelling of locations of BSs is that there will be large probability of finding two BSs quite close to each other. This will not been seen in real networks. In the scenario with a circular cell and uniform distribution of interfering BSs outside the cell seem to a more realistic, and will secure that the interfering BSs at least have a minimum distance to the serving BS. (This case is the lilac curves in the upper figures.)
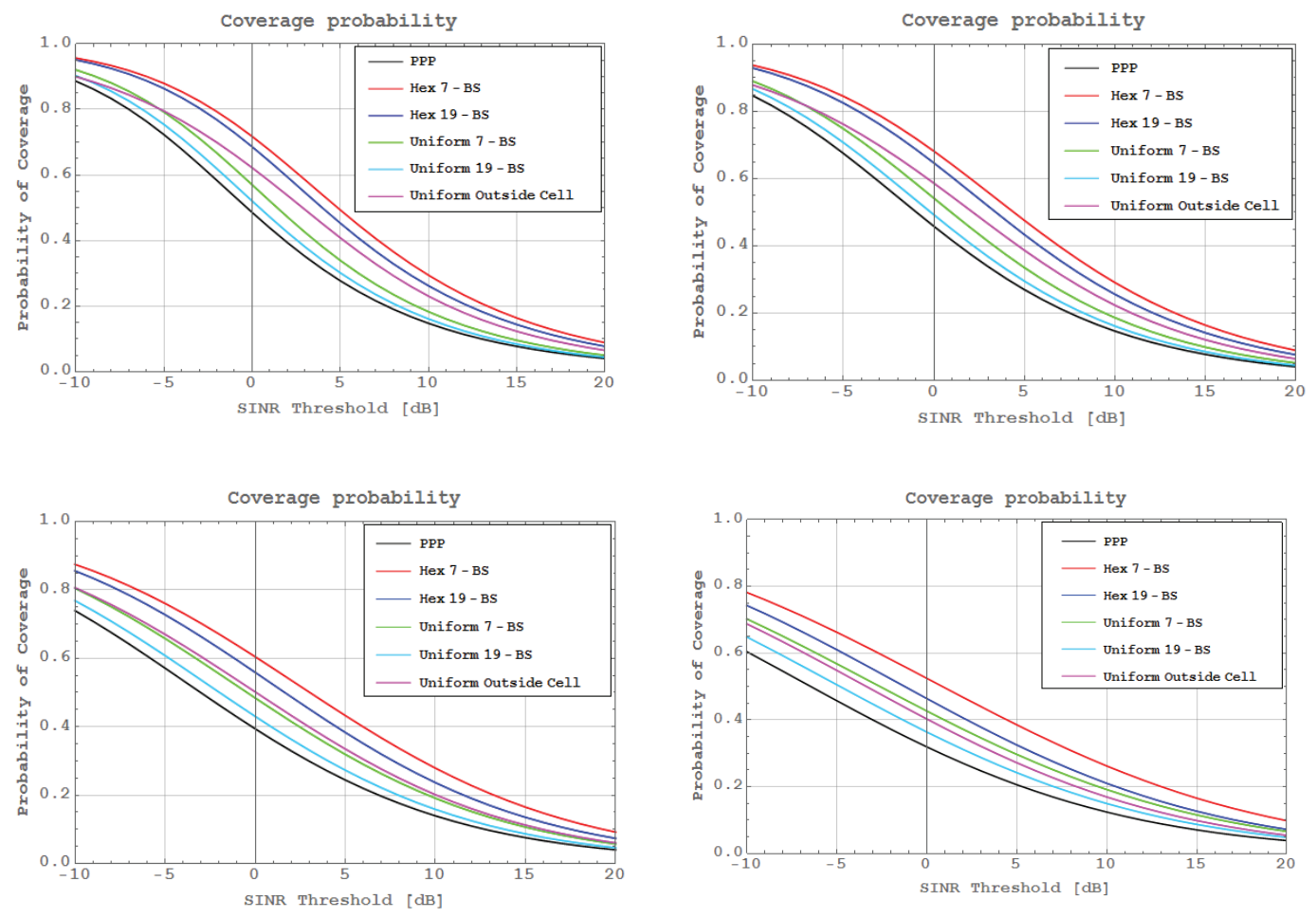

Figure 8. Coverage probabilities for hexagonal, uniform and PPP scenarios with standard deviation of shadowing: $0 \mathrm{~dB}$ top left, $3 \mathrm{~dB}$ top right, $6 \mathrm{~dB}$ bottom left and $9 \mathrm{~dB}$ bottom right.

It is possible to improve the performance compared to the PPP by just limiting the network size and spreading the BS uniformly over the area. (This corresponds to the two green curves in the 
upper diagrams.) However it might be questionable if such a reduction of the area of interfering BS will be realistic and give more accurate results. The two regular hexagonal cases, (blue and read curves in the upper diagrams) perform best. This is expected due to the regular fixed pattern where there is no possibility of having interfering BS at a closer distance than the fixed ones. Stochastic models allow for this to happen and therefore we also expect the overall interference to increase accordingly.

In Figure 9 we compare the fixed hexagonal models with the stochastic models with circular cell and where the interfering base station is uniformly located outside the cell but limited by a disc with areal that of the interfering cells in the hexagonal cases. We observe that the stochastic models give slightly poorer performance than the hexagonal models as expected. However, it turns out that the uniform placing of BSs either on a particular circle or between two circ les pretty much behaves as the fixed hexagonal cases. Especially the scenario where 6 BSs are uniformly distributed over the circumference with the same as the diameter for the hexagons (green curves) gives excellent accordance with the hexagonal 7 cell case for all value of the chosen shadowing parameter. (See the green upper curves in Figure 9 below.)
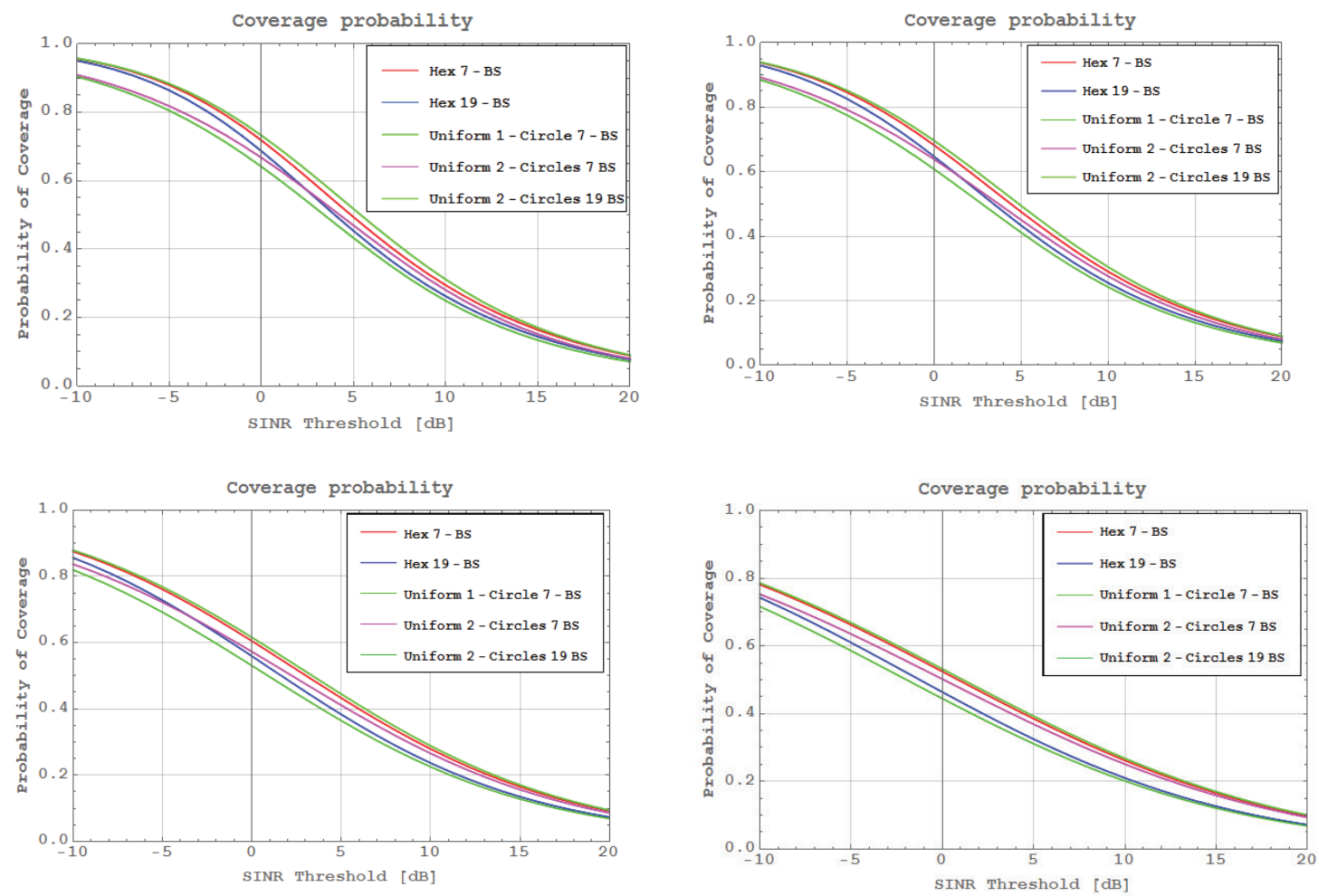

Figure 9. Coverage probabilities for comparing hexagonal scenarios with standard deviation of shadowing: $0 \mathrm{~dB}$ top left, $3 \mathrm{~dB}$ top right, $6 \mathrm{~dB}$ bottom left and $9 \mathrm{~dB}$ bottom right.

In Figure10 below we compare simulated deviated hexagonal scenarios with results from the analytical models. We consider the case where the actual location of BSs are uniformly drawn and placed in a disc surrounded the hexagons with radius of $0.9 \mathrm{~km}$ (see Figure 7 above) in the example shown in the figures. With this assumption around a quarter of the area are forbidden to put BSs, and moreover, two BSs will never be closer than at most $0.2 \mathrm{~km}$. One observation is that the deviation from the fixed hexagonal scenarios is not very big although the differences are clearly visible, but much less than for the PPP model. It is reason to believe that the deviated 
hexagonal BS model may be adapted to real deployment by adapting both the distance between hexagons and the disk radius for the deviation. For this particular case we see that the deviated hexagonal model with two rings of hexagons e.g. $19 \mathrm{BSs}$ are quite good accordance with circular cell case where the interfering BS is located uniformly outside the cell (lover green and red curves in Figure10).
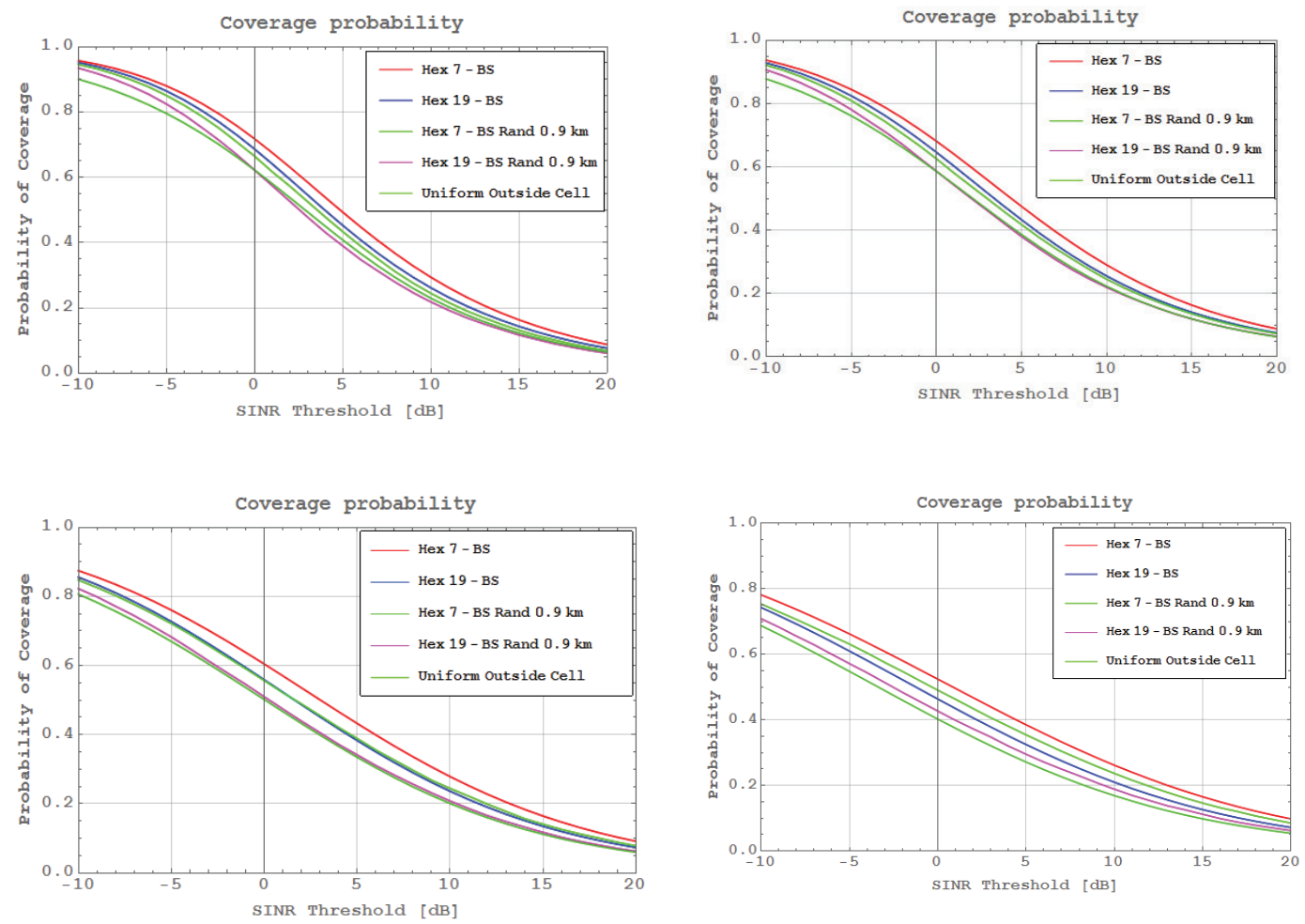

Figure10 Coverage probabilities for comparing deviated hexagonal scenarios with standard deviation of shadowing: $0 \mathrm{~dB}$ top left, $3 \mathrm{~dB}$ top right, $6 \mathrm{~dB}$ bottom left and $9 \mathrm{~dB}$ bottom right.

Another interesting observation we may draw from the scenarios is that only quite a few users will get high bitrates. For instance as seen from Figure 5 a user needs to have SINR larger than 20 $\mathrm{dB}$ to obtain normalised throughput of 5 or larger and the portion of users where this is possible is well below $20 \%$ for all the scenarios and cases considered and actual lies in the range $10-18 \%$.

\subsection{Coverage For The B-Ginibre Point Process}

Below in Figure 11, we show the coverage probability for case where the BSs are located in the plane according to a $\beta$-GPP for different parameters of the standard deviation of the shadowing. We observe that $\beta$-GPP converges to PPP case when the thinning parameter $\beta$ gets small as expected. One observation, however, is that the $\beta$-GPP modelling of BSs does not give a broad variation of the coverage probabilities in terms of the thinning parameter $\beta$ and cannot be used as a model BSs layout with quite regular layouts like classical hexagonal or deviation of hexagonal as depicted in Figure 10 above. The increase in the coverage for the $\beta$-GPP compare to PPP BS layout is quite limited as seen from the different cases shown in the figure below. Hence, we man not expect that the $\beta$-GPP will "solve" the shortcomings by the traditional PPP modelling. Another issue is the heavy computing efforts to obtain the coverage probabilities for the $\beta$-GPP. While the PPP model is fast also for shadowing parameters larger than zero, the corresponding $\beta$ GPP requires heavy computation of infinite products and sums and will typical have like 100 times larger CPU-times. 

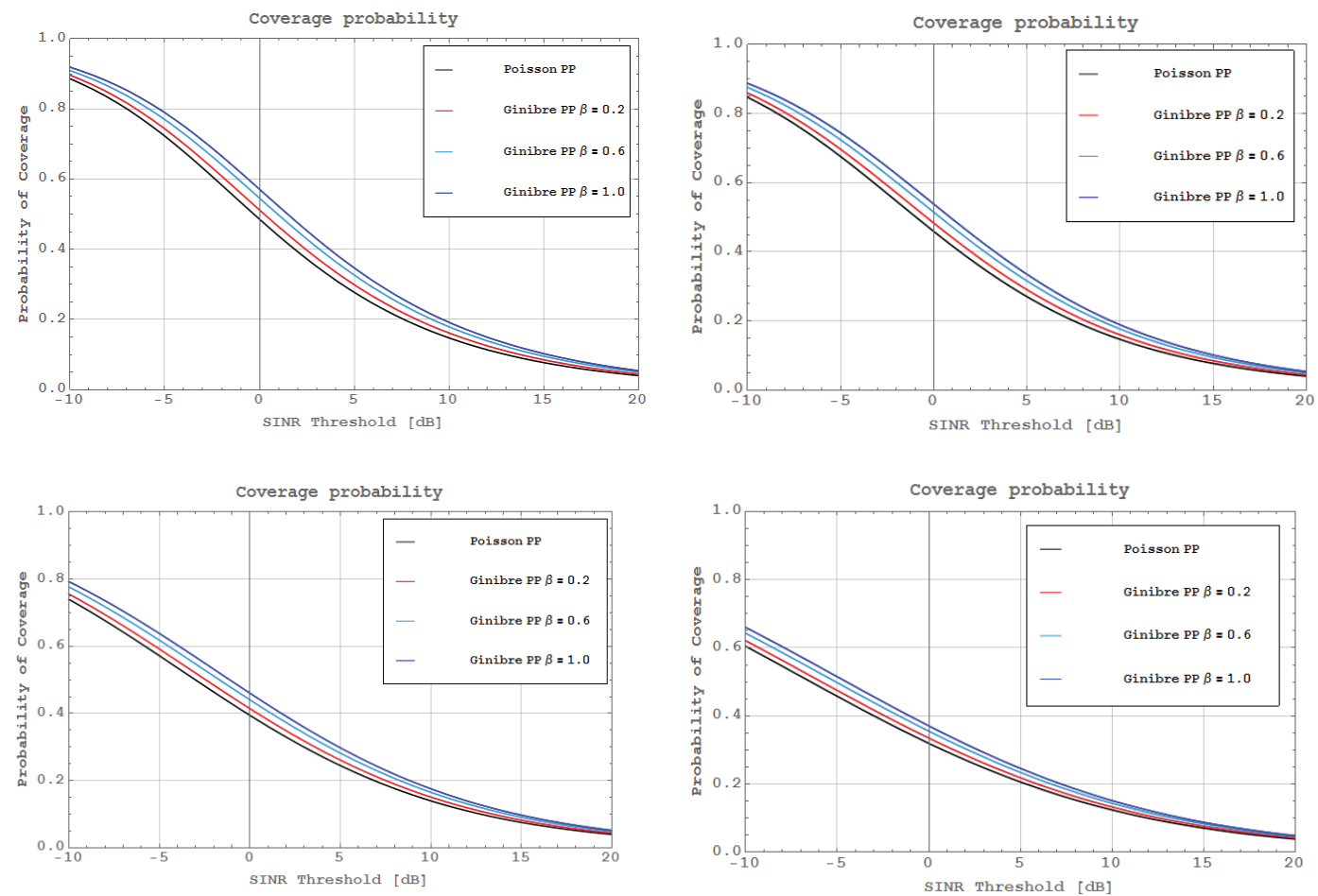

Figure 11. Coverage probabilities for Poisson PP and Ginibre PP with standard deviation of shadowing: 0 $\mathrm{dB}$ top left, $3 \mathrm{~dB}$ top right, $6 \mathrm{~dB}$ bottom left and $9 \mathrm{~dB}$ bottom right.

\subsection{SPECTRUM EFFICIENCY}

In Figure 12 we have plotted the mean obtainable spectrum efficiency for the different scenarios as a function of the standard deviation of the shadowing.

Since our examples is interference limited; i.e. it is the interference from surrounding BSs rather than the noise power that determines the SINR values we also expect the corresponding overall efficiency to be quite low. Generally we observe that the mean efficiency decrease with an increase of standard deviation of the shadowing. As expected the PPP model gives the worst performance where the mean efficiency drops from 1.09 to 0.811 for $9.0 \mathrm{~dB}$ shadowing standard deviation. For the 7 hexagonal cells scenario the corresponding decrease is from 1.83 to 1.53. All the other scenarios give values that are in between these two cases except for the scenario where 6 BSs are randomly distributed over the circumference of a circle with the same diameter as the hexagons which gives a slight higher throughput.

The $\beta$-GPP model shows similar performance for the spectrum efficiency as the PPP model but with some higher values as shown in Figure 12. For the $\beta=1$-GPP BSs layout the mean efficiency drops from 1.33 to 0.966 when the standard deviation of the shadowing increase from 0.0 for 9.0 $\mathrm{dB}$ and this is roughly an increase of around $20 \%$ compared to the PPP model. 
International Journal of Wireless \& Mobile Networks (IJWMN) Vol.9, No.3, June 2017
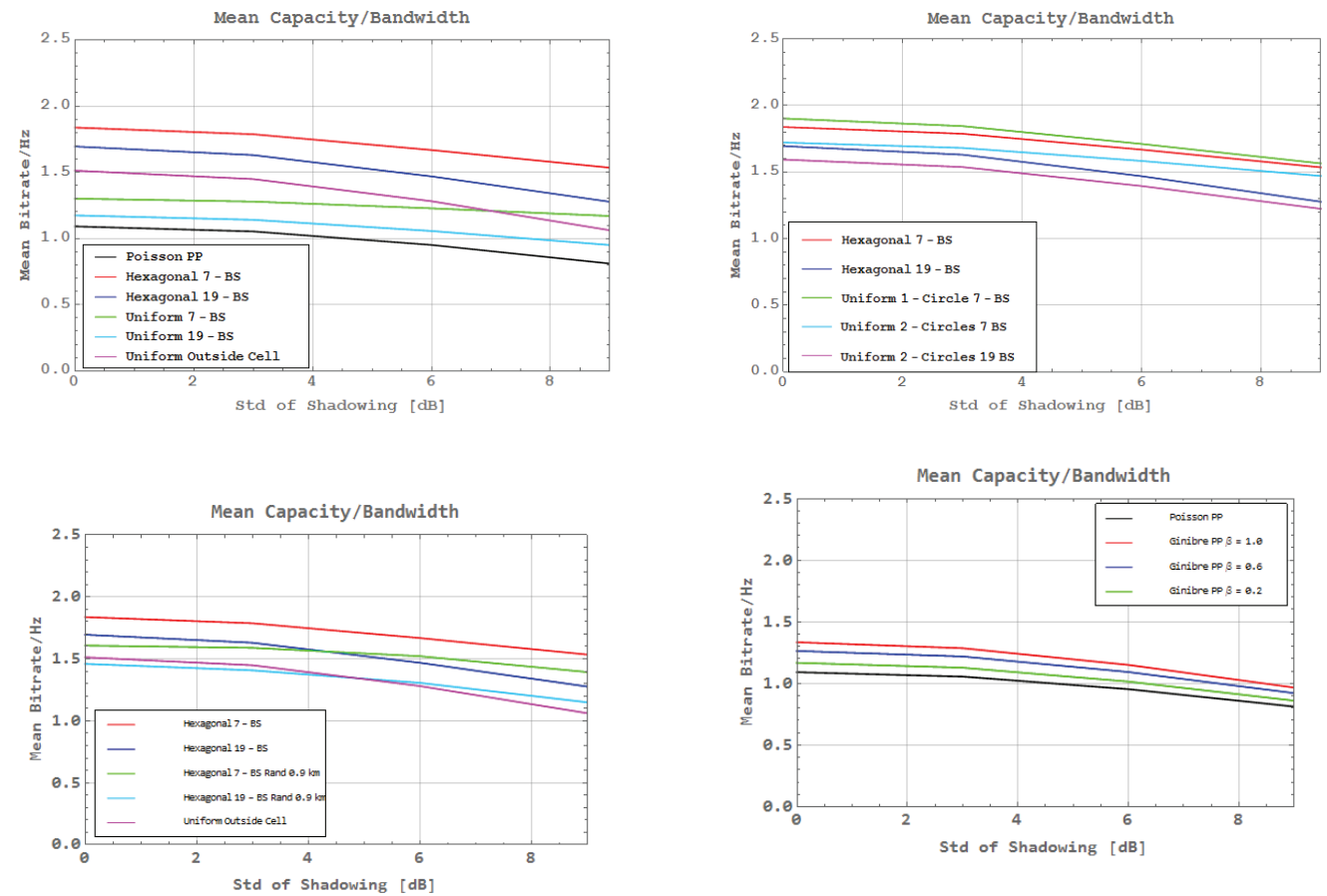

Figure 12. Mean Bitrate/Hz for the different scenarios as function of standard deviation of the shadowing.

\section{CONCLUSIONS}

In this paper we discuss and compare methods to analyse the influence of inter-cell interference have on the down link performance in cellular networks. The framework is based on classical methods to find the Laplace transform of the interference from neighbouring cells, and both fixed and random location of BSs are considered. It turns out that for Suzuki distributed fading the analysis is highly simplified, since the distribution of the SINR may be evaluated by real integral rather than a complex contour integral that would be necessary if the fading was assumed to be Log-normal. In case of Rayleigh fading only, the analysis is even more simplified.

The modelling approach allows for both fixed and stochastic locations of BSs. The stochastic models are compared with a fixed hexagonal cell layout with 7, (i.e. one ring of interfering BSs) and 19 cells, (i.e. two rings of interfering BSs). All the proposed models require quite heavily numerical computation with numerical integration of multi-dimensional integrals.

The different models are applied to quantify the effect of the interference in LTE networks where we have considered several scenarios ranging from fixed locations of BS to stochastic distribution of BSs based on PPPs. It turns out that the PPP distribution of BSs give the worst performance for the outage probabilities, while the fixed hexagonal grid with 7 or 19 BSs gives the best. The difference between these two scenarios is quite large while the scenario with uniform distribution of BS outside a circular cell gives coverage probabilities that are in between those two extremes. We believe that this model perhaps will give the best match of what is observed in real LTE networks.

When it comes to the spectrum efficiency we also see big difference between the different cases where the PPP case give only around one bit per hertz on average, while the hexagonal give nearly $50 \%$ more. We also observe a decrease in the spectrum efficiency as a function of the standard deviation of the shadowing. Hence, this shows that the popular assumption of Rayleigh faded channels only, actually will give too optimistic cell performance. 


\section{APPENDIX}

\section{Appe ndix- I. Some Properties Of The SuZuki Dis tribution}

The Suzuki distribution may be defined as the product $S=X_{\ln } X_{e}$ of a Log-normal random variable $X_{\ln }$ and an exponential random variable $X_{e}$ with mean value taken to unity. The PDF and CCDF may therefore be written as the integrals $f_{S}(x)=\int_{t=0}^{\infty} \frac{1}{t} e^{-\frac{x}{t}} L n_{\sigma}(t) d t$ and $\widetilde{F}_{S}(x)=\int_{t=0}^{\infty} e^{-\frac{x}{t}} L n_{\sigma}(t) d t$ where $L n_{\sigma}(x)$ is the Log-normal PDF. Since we have $L n_{\sigma}(t)=\frac{1}{t^{2}} \operatorname{Ln} \sigma\left(\frac{1}{t}\right)$ it is possible to express the integrals above in terms of the Laplace transform of the Log-normal distribution, and the CCDF of the Suzuki distribution is exactly the Laplace transform of the Log-normal distribution, e.g.

$$
\widetilde{F}_{S}(x)=\hat{f}_{L n_{\sigma}}(x)=\int_{t=0}^{\infty} e^{-t x} \operatorname{Ln} n_{\sigma}(t) d t
$$

For numerical calculations truncation of the integral (A-1) is done, and the error is exponentially small. If we let

$$
\widetilde{F}_{S}(x, M)=\frac{1}{\sqrt{2 \pi} \sigma} \int_{t=0}^{M} \frac{e^{-t-\frac{(\ln (t / x))^{2}}{2 \sigma^{2}}}}{t} d t
$$

then of course $\widetilde{F}_{S}(x)=\lim _{M \rightarrow \infty} \widetilde{F}_{S}(x, M)$ and further the error is exponential bounded since: $0 \leq \widetilde{F}_{S}(x)-\widetilde{F}_{S}(x, M)=\frac{1}{x} \int_{t=M}^{\infty} e^{-t} \operatorname{Ln}_{\sigma}(t / x) d t \leq e^{-M} \frac{1}{2} \operatorname{erfc}\left(\frac{\ln (M / x)}{\sigma \sqrt{2}}\right)$.

An attempt to expand the integral (A-1) in terms of the exponential function $e^{-t}=\sum_{k=0}^{\infty} \frac{(-1)^{k} t^{k}}{k !}$ yields a divergent series, however, this is not the case for the truncated integral(A-2). We find:

$$
\widetilde{F}_{S}(x, M)=\sum_{k=0}^{\infty} \frac{(-1)^{k}}{k !} x^{k} g_{k}(M / x, \sigma)
$$

where

$$
g_{k}(u, \sigma)=\int_{y=0}^{u} y^{k} L n_{\sigma}(y) d y=\frac{1}{2} e^{\frac{k^{2} \sigma^{2}}{2}} \operatorname{erfc}\left(\frac{k \sigma}{\sqrt{2}}-\frac{\ln (u)}{\sigma \sqrt{2}}\right)
$$

Note that although the series (A-3) converges for fixed truncation parameter $M$, it is not possible to interchange limit and summation in the series to obtain $\widetilde{F}_{S}(x)=\lim _{M \rightarrow \infty} \widetilde{F}_{S}(x, M)$. The reason is that the corresponding series $\sum_{k=0}^{\infty} \frac{(-1)^{k} x^{k} e^{\frac{k^{2} \sigma^{2}}{2}}}{k !}$ is divergent. Note, however, that this (divergent) series gives an excellent asymptotic expansion for $\widetilde{F}_{S}(x)$.

Similar the PDF of the Suzuki random variable is given by:

$$
f_{S}(x)=-\hat{f}_{L n_{\sigma}}^{\prime}(x)=\int_{t=0}^{\infty} t e^{-t x} \operatorname{Ln} n_{\sigma}(t) d t
$$

and now we take the corresponding truncated integral to be: 


$$
f_{S}(x, M)=\frac{1}{\sqrt{2 \pi} \sigma x} \int_{t=0}^{M} e^{-t-\frac{(\ln (t / x))^{2}}{2 \sigma^{2}}} d t
$$

For the PDF we find the following bound of the truncation error:

$0 \leq f_{S}(x)-f_{S}(x, M) \leq e^{-M} \frac{1}{\sqrt{2 \pi} \sigma} \int_{t=M / x}^{\infty} e^{-\frac{(\ln t)^{2}}{2 \sigma^{2}}} d t=e^{-M} \frac{1}{2} e^{\frac{\sigma^{2}}{2}} \operatorname{erfc}\left(\frac{\ln (M / x)}{\sigma \sqrt{2}}-\frac{\sigma}{\sqrt{2}}\right) \quad$ By expanding the integral (A-6) in terms of the exponential series as above, we now obtain a similar (convergent) series:

$$
f_{S}(x, M)=\sum_{k=0}^{\infty} \frac{(-1)^{k}}{k !} x^{k} e^{\frac{(k+1)^{2} \sigma^{2}}{2}} g_{k+1}(M / x, \sigma)
$$

where $g_{k}(u, \sigma)$ is given by $(\mathrm{A}-4)$ above.

In Appendix-III below we show that it is possible to express the CCDF of Suzuki distribution as a complex contour integral:

$$
\widetilde{F}_{S}(x)=\frac{1}{2 \pi i} \int_{\gamma_{a}} \Gamma(z) e^{-z \ln x z^{2} \sigma^{2}} \frac{2}{2} d z
$$

where $\Gamma(z)$ is the Gamma function and $\gamma_{a}=\{z=a+i y\}$ is a straight line parallel with imaginary axis with $a>0$. Differentiation now gives the PDF as:

$$
f_{S}(x)=\frac{1}{2 \pi i x} \int_{\gamma_{a}} z \Gamma(z) e^{-z \ln x+\frac{z^{2} \sigma^{2}}{2}} d z
$$

It turns out that the integral representations of $f_{S}(x)$ and $\widetilde{F}_{S}(x)$ are suitable derive approximation based on the saddle point method, e.g. as found in the book of Wong [6]. For $f_{s}(x)$ the ordinary saddle point method applies, while for $\widetilde{F}_{S}(x)$ the corresponding integral will have a simple pole at $z=0$ so for the CCDF we apply the "saddle point near a pole" method described in detail in [6].

We find the following asymptotic approximations:

$$
\begin{gathered}
f_{S}^{a p p}(x)=\frac{e^{f(r(x))}}{x \sqrt{2 \pi f^{\prime \prime}(r(x))}} \text { and } \\
\widetilde{F}_{S}^{a p p}(x)=\frac{1}{2} \operatorname{erfc}(\operatorname{sgn}(r(x)) \sqrt{-f(r(x))})+\frac{e^{f(r(x))}}{\sqrt{2 \pi}}\left(\frac{1}{r(x) \sqrt{f^{\prime \prime}(r(x))}}-\frac{\operatorname{sgn}(r(x))}{\sqrt{-2 f(r(x))}}\right)
\end{gathered}
$$

where

$$
f(z)=\log (z \Gamma(z))-z \ln x+\frac{z^{2} \sigma^{2}}{2} \text { and } f^{\prime \prime}(z)=\psi^{\prime}(z+1)+\sigma^{2}
$$

and where $r=r(x)$ is the solution of the (functional) equation

$$
f^{\prime}(r)=\psi(r+1)-\ln x+r \sigma^{2}=0 \text { for which } r>-1
$$


And further $\psi(z)=\frac{d}{d z} \log (\Gamma(z))=\frac{\Gamma^{\prime}(z)}{\Gamma(z)}$ is the Psi (Digamma) function. Note that the function $\psi(z+1)$ will have poles at $z=-1,-2,-3, \ldots$, and hence the functional equation (A-13)gives multiple roots. For the asymptotic expansion we must choose the root for which $r>-1$.

It turns out that the asymptotic approximation $\widetilde{F}_{S}^{a p p}(x)$ yields an excellent uniform approximation of the corresponding CCDF. By numerical inspection we observe that $0 \leq \widetilde{F}_{S}^{\text {app }}(x)-\widetilde{F}_{S}(x) \leq 0.006$ for all values of $\sigma$.

Also the Laplace transform of the PDF and CCDF for the Suzuki distribution is readily obtained by integrals:

$$
\hat{f}_{S}(y)=E\left[e^{-y S}\right]=\int_{t=0}^{\infty} \frac{t}{t+y} L n_{\sigma}(t) d t \text { and } \hat{F}_{S}(y)=\int_{u=0}^{\infty} e^{-y u} \widetilde{F}_{S}(u) d u=\int_{t=0}^{\infty} \frac{1}{t+y} L n_{\sigma}(t) d t
$$

Contour integral expressions for the transforms $\hat{F}_{s}(y)$ and $\hat{f}_{s}(y)$ are possible to obtain by staring with (A-8)and (A-9) respectively. By using the fact that $\int_{x=0}^{\infty} e^{-x y-z \ln x} d x=y^{-1+z} \Gamma(1-z)($ for $\operatorname{Re}(z)<1)$ and applying Euler's reflection formula $\Gamma(1-z) \Gamma(z)=\frac{\pi}{\sin (\pi z)}$ we find:

$$
\hat{F}_{S}(y)=\frac{1}{2 \pi i y} \int_{\gamma_{a}} \frac{\pi}{\sin (\pi z)} e^{z \log y+\frac{z^{2} \sigma^{2}}{2}} d z
$$

where $\gamma_{a}=\{z=a+i y\}$ is a straight line parallel with imaginary axis with $a<1$. Similar we find

$$
\hat{f}_{S}(y)=-\frac{1}{2 \pi i} \int_{\gamma_{a}} \frac{\pi}{\sin (\pi z)} e^{z \log y+\frac{z^{2} \sigma^{2}}{2}} d z
$$

where $\gamma_{a}=\{z=a+i y\}$ now taken as a straight line parallel with imaginary axis with $a<0$.

\section{APPENDIX II:Contour Integral RePRes entation Of The CCDF Of A SuZUKI DISTRIBUTED RANDOM VARIABLE}

We let the function $\Omega(x)$ be defined by the complex contour integral

$$
\Omega(x)=\frac{1}{2 \pi i} \int_{\%} \Gamma(z) e^{-z \ln x+\frac{z^{3} \sigma^{z}}{2}} d z
$$

where $\Gamma(z)$ is the Gamma function and $\gamma_{a}=\{z=a+i y\}$ is a straight line in the complex plane right to the origin, i.e. with $a>0$. By applying the integral representation of the Gamma function $\Gamma(z)=\int_{t=0}^{\infty} t^{z-1} e^{-t} d t$ (for $\left.\operatorname{Re}(z)>0\right)$, and by changing the order of integration we find $\Omega(x)=\int_{t=0}^{\infty} t^{-1} e^{-t}\left(\frac{1}{2 \pi i} \int_{\gamma_{a}} e^{z \ln (t / x)+\frac{z^{2} \sigma^{2}}{2}} d z\right) d t$. But $\frac{1}{2 \pi i} \int_{\gamma_{a}} e^{z \ln (t / x)+\frac{z^{2} \sigma^{2}}{2}} d z=\frac{e^{-\frac{\left(\ln (t / x)^{2}\right.}{2 \sigma^{2}}}}{2 \pi i} \int_{\gamma_{a}}^{\frac{\sigma^{2}}{2}\left(z+\frac{\ln (t / x)}{\sigma^{2}}\right)^{2}} d z=\frac{e^{-\frac{\left(\ln (t / x)^{2}\right.}{2 \sigma^{2}}}}{\sqrt{2 \pi} \sigma}$ which then gives $\Omega(x)=\frac{1}{\sqrt{2 \pi} \sigma} \int_{t=0}^{\infty} \frac{e^{-t-\frac{\left(\ln (t / x)^{2}\right.}{2 \sigma^{2}}}}{t} d t$ and is exactly the integral representation of the CCDF of the 
Suzuki distribution denoted $\widetilde{F}_{S}(x)$, given by (A-1) above.

In fact if we instead use the (lower) incomplete Gamma function $\gamma(z, M)=\int_{t=0}^{M} t^{z-1} e^{-t} d t$ in (A-17) the corresponding integral is found $\frac{1}{2 \pi i} \int_{\gamma_{a}} \gamma(z, M) e^{-z \ln x+\frac{z^{2} \sigma^{2}}{2}} d z=\frac{1}{\sqrt{2 \pi} \sigma} \int_{t=0}^{M} \frac{e^{-t-\frac{(\ln (t / x))^{2}}{2 \sigma^{2}}}}{t} d t=\widetilde{F}_{S}(x, M)$ where $\widetilde{F}_{S}(x, M)$ is the truncated CCDF defined by (A-2).

\section{APPENDIXIII: Some Numericalis Sues When Calculating The Dis Tribution OF SINR FOR SUZUKI DIS TRIBUTED FADING}

For numerical calculations we take the truncated version of (60) by defining:

$$
\widetilde{F}(x, M)=\frac{1}{x} \int_{y=0}^{M} H(y) L n_{\sigma}(y / x) d y=\int_{y=0}^{M / x} H(x y) L n_{\sigma}(y) d y
$$

where we assumes that $M>x$. Then of course $\widetilde{F}(x)=\lim _{M \rightarrow \infty} \widetilde{F}(x, M)$ and we easily obtain bound on the error $\operatorname{Er}(x, M)=\widetilde{F}(x)-\widetilde{F}(x, M)$ since $\operatorname{Er}(x, M) \leq h_{M} \frac{1}{x} \int_{y=M}^{\infty} \operatorname{Ln} n_{\sigma}(y / x) d y=\frac{h_{M}}{2} \operatorname{erfc}\left(\frac{\ln (M / x)}{\sqrt{2} \sigma}\right)$ where $h_{M}=\sup _{y \in[M, \infty)} H(y)$. Hence the error will be small by either choosing $M$ so large that either $h_{M}$ is small or $\frac{1}{2} \operatorname{erfc}\left(\frac{\ln (M / x)}{\sqrt{2} \sigma}\right)$ is small (or both e.g. the product).

It is possible to expand the integral in some different ways. By applying the Taylor expansion $H(y)=\sum_{k=0}^{\infty} \frac{H^{(k)}(0)}{k !} y^{k}$ yields the following series of $\widetilde{F}(x, M)$ :

$$
\widetilde{F}(x, M)=\sum_{k=0}^{\infty} \frac{H^{(k)}(0)}{k !} x^{k} g_{k}(M / x, \sigma)
$$

where $g_{k}(u, \sigma)$ is given by (A-4) in terms of the complementary error function.

Observe that it is not possible to interchange the summation and limit in (A-18) to obtain $\widetilde{F}(x)$ since the corresponding series $\sum_{k=0}^{\infty} \frac{H^{(k)}(0)}{k !} x^{k} e^{\frac{k^{2} \sigma^{2}}{2}}$ is divergent unless $\sigma=0$. (Also from (A-18) we have that $\widetilde{F}(x, M)=\sum_{k=0}^{\infty} \frac{H^{(k)}(0)}{k !} y^{k}=H(y)$ when $\sigma \rightarrow 0$ and $M>x$.)For small $\sigma$ it will be better to expand around the current value of $x$ by taking $H(x y)=\sum_{k=0}^{\infty} x^{k} \frac{H^{(k)}(x)}{k !}(y-1)^{k}$ giving:

$$
\widetilde{F}(x, M)=\sum_{k=0}^{\infty} \frac{H^{(k)}(x)}{k !} x^{k} h_{k}(M / x, \sigma)
$$

where

$$
h_{k}(u, \sigma)=\int_{y=0}^{u}(y-1)^{k} L n_{\sigma}(y) d y=\sum_{l=0}^{k}(-1)^{k-l}\left(\begin{array}{l}
k \\
l
\end{array}\right) g_{l}(u, \sigma)
$$

and where $g_{k}(u, \sigma)$ is given by (A-4). (Observe that for $u>1$ and $\sigma \rightarrow 0$ we have $h_{0}(u, \sigma) \rightarrow 1$ and $h_{k}(u, \sigma) \rightarrow 0$ for $k \geq 1$.)

An alternative to standard numerical integration of (A-17) we propose a procedure where we 
approximate $H(y)$ by piecewise linear functions in the interval $[0, M]$ and then integrate over the Lognormal distribution. For a $N$ point approximation with the points $\left\{y_{1}=0, y_{2}, \ldots, y_{N-1}, y_{N}=M\right\}$ in increasing order we find the following approximation:

$$
\begin{aligned}
& \widetilde{F}(x, M) \approx \sum_{i=1}^{N-1} \frac{1}{y_{i+1}-y_{i}}\left(x\left(H\left(y_{i+1}\right)-H\left(y_{i}\right)\right)\left(g_{1}\left(y_{i+1} / x, \sigma\right)-g_{1}\left(y_{i} / x, \sigma\right)\right)\right. \\
& \left.+\left(y_{i+1} H\left(y_{i}\right)-y_{i} H\left(y_{i+1}\right)\right)\left(g_{0}\left(y_{i+1} / x, \sigma\right)-g_{0}\left(y_{i} / x, \sigma\right)\right)\right)
\end{aligned}
$$

where $g_{k}(u, \sigma)$ is given by (A-4) in terms of the complementary error function.

\section{REFERENCES}

[1] J. G. Andrews, F. Baccelli, R. K. Ganti, A Tractable Approach to Coverage and Rate in Cellular Networks, IEEE Transaction on Communications, vol. 59, no. 11, November 2011.

[2] 3GPP TS 36.213 V9.2.0 (2010-06), Physical layer procedures, Table 7.2.3-1: 4-bit CQI Table.

[3] C. Mehlfuhrer, M. Wrulich, J. C. Ikuno, D. Bosanska, M. Rupp, Simulating the Long Term Evolution Physical Layer,17th European Signal Processing Conference (EUSIPCO 2009), Glasgow, Scotland, August 24-28, 2009.

[4] B. Sklar, Rayleigh Fading Channels in Mobile Digital Communication Systems Part I: Characterization and Part II: Mitigation, IEEE Communications Magazine, July 1997.

[5] M. Abramowitz, I. A. Stegun, Handbook of Mathematical Functions, Dover Publications, Inc., New York, 1970.

[6] R. Wong, Asymptotic Approximations of Integrals, Academic Press, Inc. San Diego, CA. 1989.

[7] H. Suzuki, "A statistical model for urban radio propagation," IEEE Transactions on Communications, vol. 25, no. 7, pp. 673 - 680, jul 1977.

[8] COST Action 231, "Digital mobile radio towards future generation systems, final report," tech. rep., EuropeanCommunities, EUR 18957, 1999.

[9] A. J. Coulson, A. G. Williamson, and R. G. Vaughan, "A statistical basis for lognormal shadowing effects in multipath fading channels", IEEE Transactions on Communications, vol. 46, no. 4, pp. 494-502, Apr. 1998.

[10] C. E. Shannon, "A mathematical theory of communication,” Bell Syst. Tech. I., vo 1. 27, pp. 379423, 623-656, July-Oct. 1948.

[11] T. K. Sarkar,Z. Ji, K. Kim, A Medouri,M. Salazar-Palma, "A Survey of Various Propagation Models for Mobile Communication”, Antennas and Propagation Magazine, Vol. 45, No. 3, pp. 51-82, June 2003.

[12] A. Goldman, "The Palm Measure and the Voronoi Tessellation for the Ginibre Process", Ammals of Applied Probability, vol. 20, no 1, 2010, pp 90-128.

[13] N. Deng, W Zhou and M. Haenggi, "The Ginibre Point Process as a Model for Wireless Network With Repulsion", IEEE Transactions on Communications, vol. 14, No 1, January 2015.

[14] I. Nakata and N. Miyoshi, "Spatial stochastic models for analysis of heterogeneous cellular networks with repulsively deployed base stations",Performance Evaluation, Volume 78, August 2014, pp. 7-17.

[15] H. S. Dhillon, R. K. Ganti,J. G. Andrews, "Load-Aware Modeling and Analysis of Heterogeneous Cellular Networks",IEEE Transactions on Wireless Communications, vol. 12, no. 4, Apr. 2013, pp 1666-1677.

[16] H. S. Dhillon, M. Kountouris, and J. G. Andrews, "Downlink MIMO HetNets: Modeling, Ordering Results and Performance Analysis",IEEE Transactions on Wireless Communications, vol. 12, no. 10, Apr. 2013, pp 5208-5222.

[17] S.Singh, H. S. Dhillon, andJ. G. Andrews, "Offloading in Heterogeneous Networks: Modeling, Analysis, and Design Insights", IEEE Transactions on Wireless Communications, vol. 12, no. 5, May 2013, pp 2484-2497.

[18] S. Mukherjee, "Distribution of Downlink SINR in Heterogeneous Cellular Networks", IEEE Journal on Selected Areas Communications, vol. 30, no. 3, Apr. 2012, pp 575-585.

[19] Y. Li, F. Baccelli, H. S. Dhillon and J. G. Andrews, "Statistical Modeling and Probabilistic Analysis of Cellular Networks with Determinantal Point Processes", submitted to IEEE Transactions on Communications, Dec. 2014. 


\section{AUTHORS}

Olav Østerbø received his MSc in Applied Mathematics from the University of Bergen in 1980 and his PhD from the Norwegian University of Science and Technology in 2004. He joined Telenor in 1980. His main interests include teletraffic modeling and performance analysis of different parts of communication networks. Activities in recent years have been related to performance analysis and QoS aspects of packet networks and modelling and capacity analysis of radio access networks like WLAN and LTE.

Ole Grøndalen received a Master of Science degree in physics from the University of Oslo in 1987. Since then he has been working with development and analysis of radio systems at Telenor Research. His current interests include Self-Organized Networks, cognitive radio and backhaul solutions.
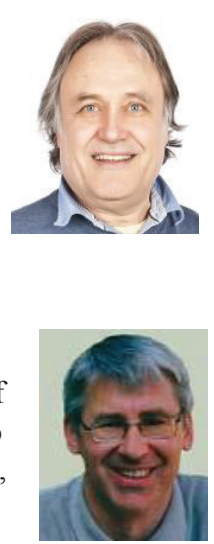